\title{
Do seismic intensity measures (IMs) measure up?
}

\author{
M. Grigoriu \\ Cornell University, Ithaca NY 14853-3501, USA \\ Phone: 607-255-3334, e-mail: mdg12@cornell.edu
}

\begin{abstract}
The assumption that demand parameters $D$ for realistic structures, i.e., complex, nonlinear dynamic systems, subjected to seismic acceleration processes $A(t)$ correlate satisfactorily with maxima $S_{a}(T)$ of responses of single degree of freedom (SDOF) linear systems to $A(t)$ is the cornerstone of current definitions of seismic intensity measures (IMs). We show that, generally, $S_{a}(T)$ and $D$ are weakly dependent and conclude that fragilities defined as functions of $S_{a}(T)$ have large uncertainties. The analysis considers linear/nonliear systems and single/multiple ordinates of $S_{a}(T)$. Tools of random vibration, copula models, and multivariate extreme value theory are employed to quantify the dependence between $S_{a}(T)$ and $D$.
\end{abstract}

Keywords: Copulas, extreme value theory, fragility, Monte Carlo simulation, seismic intensity measure, specific barrier model

\section{Introduction}

Fragilities are probabilities that structural systems enter specified damage states for given seismic intensity measures (IMs) and constitute essential tools for performance-based earthquake engineering. To be useful, IMs need to be efficient, i.e., structural demand parameters $D$ conditional on IMs have small variances, and sufficient, i.e., the distributions of the conditional random variables $D \mid$ IM are completely defined for given IMs $[4,11,12,13]$. For efficient IMs, the distribution of the conditional variables $D \mid \mathrm{IM}$ can be estimated from relatively small sets of structural responses. For sufficient IMs, the conditional random variables $D \mid$ (seismic hazard) and $D \mid \mathrm{IM}$ have similar distributions so that probability plots of structural damage versus IMs, i.e., fragilities, are meaningful.

IMs used currently in performance-based earthquake engineering are functionals of the seismic ground acceleration process $A(t)$, and can be divided in two groups. The first group includes functionals of samples of $A(t)$, e.g., the peak ground acceleration (PGA) and the peak ground velocity (PGV). The second group consists of functionals of filtered versions of samples of $A(t)$, e.g., single/multiple ordinates of the pseudo-acceleration response spectrum $S_{a}(T)$ for selected periods $T$. Our focus is on IMs in the second group since they are used extensively in practice.

(C) 2016. This manuscript version is made available under the Elsevier user license http://www.elsevier.com/open-access/userlicense/1.0/ 
Efficiency, sufficiency, and other properties of IMs have been studied extensively during the last two decades. Yet, these properties could not be assessed precisely since the distributions of IMs and demand parameters are not known due to the limited information on the seismic acceleration process $A(t)$. It has been proposed to (1) use concepts of the information theory to quantify the information carried by various IMs for selected demand parameter [4] and use it to rate their performance or (2) assess the performance of IMs for selected structural demand parameters based on benchmark studies [11, 12, 13]. These studies recognize that sufficient IMs may not exist and that resulting ratings of IMs may be affected by the considered information metrics and benchmark studies.

This paper examines critically the unstated assumption that responses of complex nonlinear structural systems can be predicted with satisfactory accuracy from those of linear SDOF systems. The assumption is the cornerstone of current definitions of IMs. We confine our analysis to seismic acceleration processes $A(t)$ with known probability law so that the joint distribution of $S_{a}(T)$ and structural demand parameters $D$ can be found. The seismological model in [16] and other models are used to characterize the seismic acceleration process $A(t)$. Let $X_{\text {sdof }}(t)$ and $X(t)$ denote responses of linear single degree of freedom (SDOF) and complex nonlinear systems subjected to $A(t)$. These responses and, therefore, $S_{a}(T)$ and $D$, cannot be independent as functionals of the same process $A(t)$. However, they are likely to be weakly dependent for realistic structures since the stochastic processes $X_{\text {sdof }}(t)$ and $X(t)$ have very different sample properties and frequency contents. Concepts of the random vibration and the multivariate extreme value theories and copula models are used to quantify the dependence between $S_{a}(T)$ and $D$.

It is found that, for realistic structural systems, (1) the dependence between $S_{a}(T)$ and $D$ is weak so that fragilities defined as functions of $S_{a}(T)$ have large uncertainties and (2) the fragilities defined as functions of multiple ordinates of $S_{a}(T)$ provide only a slight improvement over those based on single ordinates of $S_{a}(T)$. It is concluded that fragilities need to be defined as functions of parameters of the law of $A(t)$, e.g., fragility surfaces of the type introduced in [10], rather than properties of functionals of samples of this process, e.g., ordinates of the pseudo-acceleration response spectrum $S_{a}(T)$.

\section{Problem definition}

Let $P_{f}(\xi)=P(\mathcal{D} \mid \mathrm{IM}=\xi)=P(D \in I \mid \mathrm{IM}=\xi)$ denote the probability that a structural system enter a damage states $\mathcal{D}$ if subjected to ground motions with scalar/vectorvalued intensity measure $\xi$, where $I$ defines the set of demand parameters which yield damage state $\mathcal{D}$. Fragilities are plots of $P_{f}(\xi)$ against $\xi$. Generally, the probabilities $P_{f}(\xi)$ are estimated from structural responses to seismic records scaled in some manner [3] so that their accuracy depends on the sample size, scaling procedure, and properties of IMs.

Suppose the seismic ground acceleration at a site can be modeled by a stochastic process $A(t), t \in[0, \tau]$. Let $X_{\text {sdof }}(t)$ and $X(t)$ denote the response of a single degree of freedom (SDOF) linear oscillator with damping ratio $\zeta$ and period $T$ and the response of an arbitrary structural system subjected to the same ground acceleration $A(t)$. Generally, $X(t)$ is a vector-valued process. For simplicity, we consider real-valued demand parameters of the type $D=\max _{0 \leq t \leq \tau}|h(X(t))|$, where $\tau$ denotes the duration of the seismic event and $h$ maps $\mathrm{X}(\mathrm{t})$ 
into a real-valued response of interest, e.g., an interstory displacement or a floor acceleration. The IM of interest is the pseudo-spectral acceleration $S_{\mathrm{a}}(T)=(2 \pi / T)^{2} \max _{0 \leq t \leq \tau}\left|X_{\text {sdof }}(t)\right|$. The input-output mappings $A(t) \mapsto X_{\text {sdof }}(t) ; X(t) \mapsto S_{a}(T) ; D$ show that $S_{a}(T)$ and $D$ are dependent random variables as functionals of $A(t), 0 \leq t \leq \tau$.

Intuition suggests that $S_{a}(T)$ and $D$ are weakly dependent since they are obtained from the stochastic processes $X_{\text {sdof }}(t)$ and $X(t)$ which have very different properties as solutions of simple linear and complex nonlinear random vibration problems to $A(t)$. For example, if $A(t)$ is Gaussian, $X_{\text {sdof }}(t)$ and $X(t)$ are Gaussian and non-Gaussian processes with very different frequency bands. If this intuition is correct, fragilities defined as functions of single/multiple ordinates of $S_{a}(T)$ have significant uncertainties so that they are of limited practical use. The main objective of this study is to quantify the dependence between $S_{a}(T)$ and $D$ and determine implicitly whether fragilities defined as functions of current IMs provide useful information for performance-based earthquake engineering.

To achieve this objective, we quantify the dependence between $S_{a}(T)$ and $D$ by using a broad range of statistical tools, which are discussed in Sect. 3. If the dependence between $S_{a}(T)$ and $D$ is very strong and weak, then $S_{a}(T)$ is a very good and unsatisfactory IM. Corresponding fragilities plotted against $S_{a}(T)$ are informative and provide at best limited information, respectively.

The remainder of this section illustrates the relationship between $S_{\mathrm{a}}(T)$ and $D$ for earthquakes of increasing intensities, outlines our formulation, and discusses briefly the computational tools used in analysis.

\subsection{An illustration}

Suppose $X(t)$ is the displacement of a Duffing oscillator with parameters $\left(\nu_{0}, \zeta, \beta\right)$ which is at rest at the initial time and is subjected to a ground acceleration process $A(t)$. Then, $X(t)$ satisfies the differential equation

$$
\ddot{X}(t)+2 \zeta \nu_{0} \dot{X}(t)+\nu_{0}^{2}\left(X(t)+\beta X(t)^{3}\right)=-A(t), \quad t \in[0, \tau],
$$

with initial conditions $X(0)=0$ and $\dot{X}(0)=0$. If $\beta=0$ and $\nu_{0}=2 \pi / T$, then $X(t)=$ $X_{\text {sdof }}(t)$ is the displacement of a linear oscillator with damping ratio $\zeta$ and period $T$. Otherwise, $X(t)$ is the response of a simple oscillator with cubic nonlinearity. The random variables $S_{\mathrm{a}}(T)=(2 \pi / T)^{2} \max _{0 \leq t \leq \tau}\left|X_{\text {sdof }}(t)\right|$ and $D=\max _{0 \leq t \leq \tau}|X(t)|$ are dependent since they are functionals of the same input, the seismic ground acceleration process $A(t)$.

The dependence between $S_{\mathrm{a}}(T)$ and $D$ varies with the intensity of the ground motion and the magnitude of the nonlinear stiffness component. For small seismic excitation, the contribution of the cubic nonlinearity $\nu_{0}^{2} \beta X(t)^{3}$ to the displacement $X(t)$ of the Duffing oscillator is insignificant so that $X(t)$ should be similar to the displacement $X_{\text {sdof }}(t)$ of the associate linear oscillator $(\beta=0)$. In this case, the dependence between $S_{\mathrm{a}}(T)$ and $D$ is expected to be strong so that $S_{\mathrm{a}}(T)$ is a very good IM. For large seismic excitations, the cubic nonlinearity $\nu_{0}^{2} \beta X(t)^{3}$ contributes to $X(t)$ so that both the frequency contents and the distributions of $X(t)$ and $X_{\text {sdof }}(t)$ differ. For example, if $A(t)$ is a Gaussian process, then $X_{\text {sdof }}(t)$ and $X(t)$ are Gaussian and non-Gaussian processes. In this case, the correlation between $S_{\mathrm{a}}(T)$ and $D$ is expected to be weaker so that $S_{a}(T)$ is a less satisfactory IM. 
These observations are consistent with the numerical results in Fig. 1 which show $n=$ 500 independent samples of the random vector $\left(S_{a}(T) /(2 \pi / T)^{2}, D\right)$ for a Duffing oscillator


Figure 1: Scatter plots of $n=500$ independent samples of $\left(S_{a}(T) /(2 \pi / T)^{2}, D\right)$ for $\beta=3$ and a stationary Gaussian BLWN $A(t)$ with mean 0 , variance 1 , and frequency band $[0,10]$ scaled by 1, 5, and 10 (left, middle, and right panels)

with $\nu_{0}=2 \pi, \zeta=0.05$, and $\beta=3$ that is subjected to a stationary Gaussian band-limited white noise (BLWN) $A(t)$ with mean 0 , variance 1 , and frequency band $[0,10]$ during the time interval $[0,20]$. The system is at rest at the initial time. The left, middle, and right panels are for ground accelerations $A(t)$ scaled by 1, 5, and 10. For small ground excitations corresponding to a scale factor of 1 (left panel), the dependence between $S_{a}(T) /(2 \pi / T)^{2}$ and $D$ is nearly perfect (the estimated correlation coefficient is almost 1 ). The differences between the responses $X(t)$ and $X_{\text {sdof }}(t)$ are negligible. For large ground excitations corresponding to a scale factor of 10 (right panel), the dependence between $S_{a}(T) /(2 \pi / T)^{2}$ and $D$ is weaker (the estimated correlation coefficient is 0.8107 ). The middle panel corresponds to moderate earthquakes, the scale factor is 5 . It represents a transition between the extreme cases in the left and right panels.

The plots in Fig. 1 show, in agreement with findings in [10], that $S_{a}(T)$ can be viewed as a satisfactory IM for the Duffing oscillator. We attribute this performance to the fact that the Duffing oscillator is a conservative SDOF structure whose stiffness is a perturbation of the stiffness of the associated linear $\operatorname{SDOF}(\beta=0)$ and matches the stiffness of this system for small displacements. Yet, even in this very favorable setting, the predictive capability of $S_{a}(T)$ decreases with the magnitude of the structural responses. For example, the correlation coefficients between the top $m$ samples of the demand parameter $D$ (scale factor 10) and the corresponding samples of $S_{a}(T)$ are $0.8107,0.7684,0.6418,0.4381$, and 0.3408 for $m=10,000,9,000,1,200,600$, and 150 .

\subsection{Fragility estimates}

Suppose the event $\{D \in I\}$ defines a damage state $\mathcal{D}$ for a structural system, where the interval $I$ of the real line gives the range of a demand parameter $D$ associated with $\mathcal{D}$. Fragilities are plots of the probabilities $P_{f}(\xi)=P(\mathcal{D} \mid \mathrm{IM}=\xi)=P(D \in I \mid \mathrm{IM}=\xi)$ against values $\xi$ of an IM.

The following three-step approach is commonly used to construct fragilities. First, a finite set of intensity measures $\left\{\xi_{k}\right\}$ is selected. Second, finite sets of seismic acceleration records are associated with each intensity measure $\left\{\xi_{k}\right\}$ by the following procedure. The 
available seismic records are scaled to have the selected IMs and subsets of these scaled records are associated with each $\xi_{k}$ via an optimality criterion [3]. Third, fragilities are estimated by $\hat{P}_{f}\left(\xi_{k}\right)=\sum_{j=1}^{n_{k}} 1\left(d_{k, j} \in I\right) / n_{k}$, where $1(\cdot)$ denotes the indicator function, $n_{k}$ is the number of seismic records selected for the intensity measure $\xi_{k}$, and $\left\{d_{k, j}\right\}, j=1, \ldots, n_{k}$, denote demand parameters corresponding to the seismic records selected for this IM. The set of probabilities $\left\{\hat{P}_{f}(\xi)\right\}$ viewed as functions of $\xi$ defines the fragility for damage state $\mathcal{D}$.

The accuracy of the resulting fragility estimates $\hat{P}_{f}(\xi)$ is difficult to assess since it depends in complex manner on the sample size $\left\{n_{k}\right\}$, the procedure used to select seismic records, and the effects of scaling seismic records. For example, scaling changes the statistics of the original records and yields records which are inconsistent with the observed relationship between the frequency content of seismic records and earthquake magnitudes. Also, $S_{a}(T)$ increases linearly with the scaling factor while responses of nonlinear structures do not.

We note that the only source of error in our setting relates to properties of IMs since the law of the seismic acceleration process $A(t)$ is known. The fragility is

$$
P_{f}(\xi)=P(D \in I \mid \mathrm{IM}=\xi)=E[1(D \in I \mid \mathrm{IM}=\xi)]=\int_{I} f_{D \mid \mathrm{IM}}(x \mid \xi) d x
$$

where $f_{D \mid \mathrm{IM}}(\cdot \mid \xi)$ denotes the density of the conditional variable $D \mid(\mathrm{IM}=\xi)$. It can be obtained from the above formula by integration or from the estimate $\sum_{i=1}^{n} 1\left(d_{i} \in I\right) / n$, where $\left\{d_{i}\right\}, i=1, \ldots, n$, are independent samples of $D$. If the dependence between $D$ and IM is strong, the random variable $D \mid \mathrm{IM}$ has small variance and the conditional density $f_{D \mid \mathrm{IM}}(\cdot \mid \xi)$ is concentrated about its expectation. This density becomes a $\delta$-function in the limit as $D$ and IM are perfectly dependent. The opposite holds for weakly dependent demand parameters and IMs. In this case, the random variable $D \mid \mathrm{IM}$ has large variance and corresponding fragilities, which are expectations (see Eq. 2), may yield only course information on structural performance. In the limit as $D$ and IM are independent, the random variables $D \mid \mathrm{IM}$ and $D$ are equal in distribution so that the fragility $P_{f}(\xi)=\int_{I} f_{D}(x) d x$ does not depend on $\xi$.

\section{Dependence metrics}

Our main objective is to determine weather fragilities defined as functions of current IMs characterize accurately the performance of structural systems subjected to earthquakes. To achieve this objective, we examine the dependence between structural demand parameters and IMs, i.e., the random variables $D$ and $S_{a}(T)$, by using three statistical tools: correlation coefficients, copula models (CMs), and concepts of the multivariate extreme value theory (MEVT). This section presents essential features and limitations of these tools. The subsequent section quantifies the dependence between $D$ and $S_{a}(T)$ for a few dynamic systems by using correlation coefficients, CMs, and MEVT-based estimates.

\subsection{Correlation coefficients}

Correlation coefficients are attractively simple but rather crude metrics for the dependence between random variables. We illustrate some of the limitations of the correlation coefficients by the following examples. 
Suppose $X \sim N(0,1)$ is a standard Gaussian variable with mean 0 and variance 1 . The non-Gaussian random variable $Y=X^{2}-1$ has mean 0 and variance 2. The Pearson correlation coefficient of $X$ and $Y$ is proportional to $E[X Y]=E\left[X^{3}\right]-E[X]=0$ so that $X$ and $Y$ are said to be uncorrelated. Yet, $\operatorname{Var}[Y \mid X]=0$ since, given $X, Y$ is known. Other correlation models also view $X$ and $Y$ as nearly uncorrelated, e.g., estimates of the Spearman and Blomqvis correlation coefficients [1] based on 100,000 independent samples of $X$ are 0.0062 and 0.0014 .

Correlation coefficients may also provide misleading information on the dependence between Gaussian variables if the focus is on large values of these variables. Let $X=$

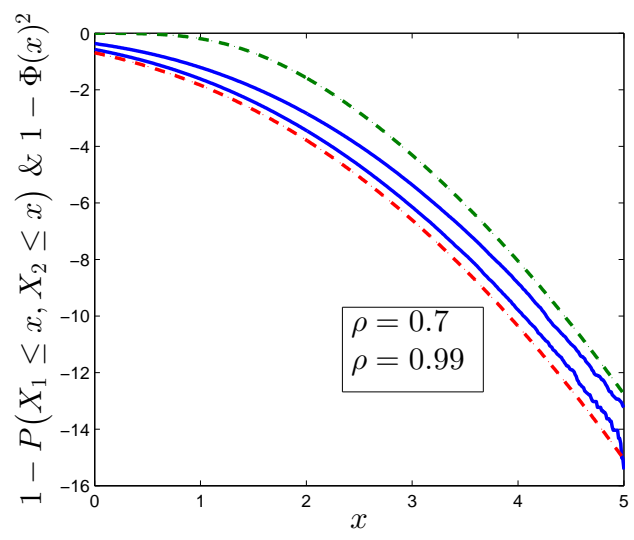

Figure 2: Probabilities $1-P\left(\cap_{i=1}^{d}\left\{X_{i} \leq x\right\}\right), 1-\Phi(x)^{d}$, and $1-\Phi(x)$ for $d=10$

$\left(X_{1}, \ldots, X_{d}\right)$ be an $d$-dimensional Gaussian vectors with equally correlated, standard components, i.e., $E\left[X_{i}\right]=0, E\left[X_{i}^{2}\right]=1$, and $E\left[X_{i} X_{j}\right]=\rho, i \neq j$. According to the normal comparison lemma [14] (Corollary 4.2.4), we have

$$
\left|P\left(\cap_{i=1}^{d}\left\{X_{i} \leq x\right\}\right)-\Phi(x)^{d}\right| \sim O\left(\exp \left(-\alpha x^{2}\right)\right)
$$

where $\Phi$ denotes the distribution of the standard Gaussian variable and $\alpha>0$ is a constant. The discrepancy between the joint distribution $P\left(\cap_{i=1}^{d}\left\{X_{i} \leq x\right\}\right)$ of $X$ and the distribution $\Phi(x)^{d}$ of an $d$-dimensional Gaussian vector $X_{\text {ind }}$ with independent, standard components vanishes as $x \rightarrow \infty$ for $|\rho|<1$. This means that extremes of the components of $X$ are asymptotically independent irrespective of their correlation. The dash lines in Fig. 2 are the probabilities $1-\Phi(x)^{d}$ and $1-\Phi(x), d=10$, i.e., the probabilities $1-P\left(\cap_{i=1}^{d}\left\{X_{i} \leq x\right\}\right)$ for $X$ with independent and perfectly correlated components. The upper and lower solid lines are estimates of the probabilities $1-P\left(\cap_{i=1}^{d}\left\{X_{i} \leq x\right\}\right)$ for $\rho=0.7$ and $\rho=0.99$. They approach $1-\Phi(x)^{d}$ as $x$ increases with rates of convergence that depend on the correlation coefficient $\rho$.

These examples show that correlation coefficients may fail to capture the dependence between non-Gaussian variables and between Gaussian variables if the focus is on simultaneously large values of these variables. These observations suggest that correlation coefficients may only provide qualitative descriptions of the dependence between $S_{a}(T)$ and $D$. 


\subsection{Copula models}

Copulas are models for the joint distributions of random vectors with arbitrary marginal distributions. They have been used extensively in applications, see [1] (Chap. 5), [5], and cited references. Copula models are conceptually simple and can be calibrated to observations by using familiar statistical tolls [5].

However, the usefulness of copulas is not universally accepted. It is argued in [15] that copulas present no particular advantage relative to a direct use of models for multivariate distributions, cannot be used to characterize joint extremes of random variables, and may misrepresent the dependence between the components of random vectors. The latter limitation relates to the two-step approach for fitting copulas to observations which separates the calibration of the dependence function, i.e., the functional form of postulated copula models, from that of the marginal distributions. Two versions of this approach are discussed later in this section.

\subsubsection{Generalities}

Let $X=\left(X_{1}, \ldots, X_{d}\right)$ be a $d$-dimensional random vector with joint distribution $F(x)=$ $P\left(X_{1} \leq x_{1}, \ldots, X_{d} \leq x_{d}\right), x=\left(x_{1}, \ldots, x_{d}\right) \in \mathbb{R}^{d}$, and marginal distributions $\left\{F_{i}(\xi)=\right.$ $\left.P\left(X_{i} \leq \xi\right)\right\}, i=1, \ldots, d$, assumed to be continuous. The components of the $d$-dimensional random vector

$$
U=\left(U_{1}, \ldots, U_{d}\right)=\left(F_{1}\left(X_{1}\right), \ldots, F_{d}\left(X_{d}\right)\right)
$$

are uniformly distributed. The copula $C:[0,1]^{d} \rightarrow[0,1]$ of $X$ is the joint distribution of $U$, i.e.,

$$
C\left(u_{1}, \ldots, u_{d}\right)=P\left(U_{1} \leq u_{1}, \ldots, U_{d} \leq u_{d}\right)=P\left(X_{1} \leq F_{1}^{-1}\left(u_{1}\right), \ldots, X_{d} \leq F_{d}^{-1}\left(u_{1}\right)\right),
$$

where the latter equality holds if the mappings $U_{i} \mapsto F_{i}\left(X_{i}\right), i=1 \ldots, d$, are bijections. In summary, copulas $C:[0,1]^{d} \rightarrow[0,1]$ are joint distributions on the unit cube $[0,1]^{d}$ with uniform marginals so that (1) $C\left(u_{1}, \ldots, u_{i-1}, 0, u_{i+1}, \ldots, u_{d}\right)=0$ and $C\left(1, \ldots, 1, u_{i}, 1, \ldots, 1\right)=$ $u_{i}$ for all $i=1, \ldots, d$, and $(2) \int_{B} d C(u) \geq 0$ on any rectangle $B \subset[0,1]^{d}$. We also note that the inequalities $\max \left\{1-d+\sum_{i=1}^{d} u_{i}, 0\right\} \leq C\left(u_{1}, \ldots, u_{d}\right) \leq \min \left\{u_{1}, \ldots, u_{d}\right\}$ hold and that finite sums $\sum_{k} \lambda_{k} C_{k}\left(u_{1}, \ldots, u_{d}\right)$ of copulas $\left\{C_{k}\right\}$ are copulas provided $\lambda_{k} \geq 0$ and $\sum_{k} \lambda_{k}=1$.

For two-dimensional copulas $C:[0,1]^{2} \rightarrow[0,1]$ these conditions are $(1) C(u, 0)=$ $C(0, v)=0, C(u, 1)=u$ and $C(1, v)=v$ for $u, v \in[0,1]$, and (2) $C\left(u_{2}, v_{2}\right)-C\left(u_{2}, v_{1}\right)-$ $C\left(u_{1}, v_{2}\right)+C\left(u_{1}, v_{1}\right) \geq 0$ for all $u_{1}, u_{2}, v_{1}, v_{2} \in[0,1]$ such that $u_{1} \leq u_{2}$ and $v_{1} \leq v_{2}$. The second requirement constitutes the finite difference version of the condition $\partial^{2} C(u, v) / \partial u \partial v \geq 0$ and implies $\int_{B} d C(u) \geq 0$.

The Sklar theorem ([1], Chap. 5) connects the joint distributions of random vectors to copulas. It states that (1) every distribution $F$ of a random vector $X=\left(X_{1}, \ldots, X_{d}\right)$ admits the representation

$$
F\left(x_{1}, \ldots, x_{d}\right)=C\left(F_{1}\left(x_{1}\right), \ldots, F_{d}\left(x_{d}\right)\right), \quad x=\left(x_{1}, \ldots, x_{d}\right) \in \mathbb{R}^{d},
$$

where $C:[0,1]^{d} \rightarrow[0,1]$ is a copula, and (2) copulas are unique on the cartesian product $R=$ $\times_{i=1}^{d} \operatorname{Ran}\left(F_{i}\right)$ of the ranges $\left\{\operatorname{Ran}\left(F_{i}\right)\right\}$ of the marginal distributions of $X$, which implies that 
the copula is unique if the marginal distributions of $X$ are continuous. Also, given a copula $C:[0,1]^{d} \rightarrow[0,1]$ and marginal distributions $\left\{F_{i}\right\}, i=1, \ldots, d$, then $C\left(F_{1}\left(x_{1}\right), \ldots, F_{d}\left(x_{d}\right)\right)$ is a $d$-dimensional joint distribution.

A large catalog of copulas is available. Gaussian and Archimedean copulas are briefly presented since they are frequently used in applications. The Gaussian copula has the form

$$
C\left(u_{1}, \ldots, u_{d}\right)=\Phi_{d}\left(\Phi^{-1}\left(u_{1}\right), \ldots, \Phi^{-1}\left(u_{d}\right)\right)=\Phi_{d}\left(\Phi^{-1}\left(F_{1}\left(x_{1}\right)\right), \ldots, \Phi^{-1}\left(F_{d}\left(x_{d}\right)\right)\right)
$$

for $u=\left(u_{1}, \ldots, u_{d}\right) \in[0,1]^{d}$ and $x=\left(x_{1}, \ldots, x_{d}\right) \in R=\times_{i=1}^{d} \operatorname{Ran}\left(F_{i}\right)$, where $\Phi_{d}$ denotes the joint distribution of an $d$-dimensional Gaussian vector $G=\left(G_{1}, \ldots, G_{d}\right)$ with specified covariance matrix and components with zero means and unit variances. Since $F_{i}\left(X_{i}\right)=$ $\Phi\left(G_{i}\right), i=1, \ldots, d$, we have

$$
P\left(\cap_{i=1}^{d}\left\{X_{i} \leq x_{i}\right\}\right)=P\left(\cap_{i=1}^{d}\left\{F_{i}^{-1}\left(\Phi\left(G_{i}\right)\right) \leq x_{i}\right\}\right)=P\left(\cap_{i=1}^{d}\left\{G_{i} \leq \Phi^{-1}\left(F_{i}\left(x_{i}\right)\right)\right\}\right),
$$

i.e., the copula model in Eq. 6 for $u_{i}=F_{i}\left(x_{i}\right), i=1, \ldots, d$. Note also that the Gaussian copula coincides with the translation random vector model in [8] (Chap. 3). Of the Archimedean copulas, frequently used two-dimensional copulas have the form

$$
\begin{aligned}
C(u, v) & =\frac{u v}{1-\theta(1-u),(1-v)}, \quad-1 \leq \theta<1, \\
C(u, v) & \left.=\left[\max \left\{u^{-\theta}+v^{-\theta}-1,0\right\}\right]^{-1 / \theta}, \quad-1 \leq \theta<\infty\right) \backslash\{0\}, \text { and } \\
C(u, v) & =\exp \left[-\left((-\log (u))^{\theta}+(-\log (v))^{\theta}\right)^{1 / \theta}\right], \quad 1 \leq \theta<\infty
\end{aligned}
$$

The second model is referred to as the Clayton copula. The limit case $\theta=0$ corresponds to a vector $X$ with independent components.

\subsubsection{Fitted copulas}

Properties of copulas are well-established and discussed extensively in the literature. Yet, the construction of copulas for $d$-dimensional random vectors $X$ from independent samples of these vectors poses difficulties since the copula-based approximations of the joint distributions of $X$ are sensitive to the form of the mapping $u \mapsto C(u)$ and the marginal distributions $\left\{F_{i}\right\}$ of $X$.

Suppose $n$ independent samples $x^{(k)}=\left(x_{1}^{(k)}, \ldots, x_{d}^{(k)}\right), k=1, \ldots, n$, of an $d$-dimensional random vector $X$ are available. We describe two methods for calibrating copulas to samples of $X$ which are commonly used in applications. For extensive discussions on this topic, including the accuracy of copula models, see $[1,5,6]$. The first method is parametric and involves two steps. First, parametric families of copulas and marginal distributions, $\left\{C\left(u ; \theta_{0}\right)\right\}$ and $\left\{F_{i}\left(x_{i} ; \theta_{i}\right)\right\}, i=1, \ldots, d$, indexed by $\theta_{0}$ and $\left\{\theta_{i}\right\}$ are postulated. It is assumed that the postulated copulas admit densities $\left\{c\left(u ; \theta_{0}\right)=\partial^{d} C\left(u ; \theta_{0}\right) / \partial u_{1} \cdots \partial u_{d}\right\}$ for each $\theta_{0}$. Second, the vector $\theta=\left(\theta_{0}, \theta_{1}, \ldots, \theta_{d}\right)$ of unknown parameters in the definition of the postulated models is characterized by the log-likelihood function

$$
\ell\left(\theta \mid x^{(k)}, k=1, \ldots, n\right)=\sum_{k=1}^{n} \log \left[c\left(F_{1}\left(x_{1}^{(k)} ; \theta_{1}\right), \ldots, F_{d}\left(x_{d}^{(k)} ; \theta_{d}\right) ; \theta_{0}\right)\right] .
$$


This function can be used to find the maximum likelihood estimate (MLE) $\hat{\theta}$ of $\theta$, which introduced in Eq. 5 yields the copula-based model

$$
\hat{F}(x)=C\left(F_{1}\left(x_{1} ; \hat{\theta}_{1}\right), \ldots, F_{d}\left(x_{d} ; \hat{\theta}_{d}\right) ; \hat{\theta}_{0}\right), \quad x=\left(x_{1}, \ldots, x_{d}\right) \in \mathbb{R}^{d},
$$

for the distribution of $X$. We also note that the likelihood function in Eq. 8 can be used to construct posterior distributions for $\theta$ if the analysis uses the Bayesian framework.

The second method is semi-parametric and also involves two steps. First, a parametric family $\left\{C\left(u ; \theta_{0}\right)\right\}$ of copulas with densities $\left\{c\left(u ; \theta_{0}\right)=\partial^{d} C\left(u ; \theta_{0}\right) / \partial u_{1} \cdots \partial u_{d}\right\}$ is postulated as in the previous method. Second, empirical distributions $\left\{\hat{F}_{i}\right\}$, rather than postulated parametric models, are used for the unknown marginal distributions $\left\{F_{i}\right\}$ of $X$ to construct the log-likelihood function of $\theta_{0}$. The corresponding log-likelihood function has the form

$$
\begin{aligned}
\ell\left(\theta_{0} \mid x^{(k)}, k=1, \ldots, n\right) & =\sum_{k=1}^{n} \log \left[c\left(\hat{F}_{1}\left(x_{1}^{(k)}\right), \ldots, \hat{F}_{1}\left(x_{d}^{(k)}\right) ; \theta_{0}\right)\right. \\
& =\sum_{k=1}^{n} \log \left[c\left(r_{1}^{(k)} /(n+1), \ldots, r_{d}^{(k)} /(n+1) ; \theta_{0}\right)\right]
\end{aligned}
$$

where $\hat{F}_{i}(\xi)=\sum_{s=1}^{n} 1\left(x_{i}^{(s)} \leq \xi\right) /(n+1)$ and $r_{i}^{(k)}=\sum_{s=1}^{n} 1\left(x_{i}^{(s)} \leq x_{i}^{(k)}\right)$ denote the empirical distribution of $X_{i}$ and the rank of observation $k$ for component $i=1, \ldots, d$ in ascending order [5]. Note that, if $x_{i}^{\left(k_{0}\right)}$ is the smallest or the largest observation for component $i$, its rank is $r_{i}^{\left(k_{0}\right)}=1$ or $r_{i}^{\left(k_{0}\right)}=n$. The second equality in Eq. 10 holds since $(n+1) \hat{F}_{i}\left(x_{i}^{(k)}\right)$ gives the number of observations of $X_{i}$ smaller that $x_{i}^{(k)}$, which coincides with the rank $r_{i}^{(k)}$ of observation $x_{i}^{(k)}$. The scaling $1 /(n+1)$, rather than $1 / n$, is used to avoid dealing with points on the boundary of the unit cube $[0,1]^{d}[6]$. The copula-based estimate of the joint distribution of $X$ has the form

$$
\hat{F}\left(x_{1}, \ldots, x_{d}\right)=C\left(\hat{F}_{1}\left(x_{1}\right), \ldots, \hat{F}_{d}\left(x_{d}\right) ; \hat{\theta}_{0}\right),
$$

where $\hat{\theta}_{0}$ denotes the MLE of $\theta_{0}$.

The parametric models used for copulas are essential for the construction of realistic representations of the distribution of $X$ since their functional form determines the dependence structure of this vector. The empirical copula,

$$
\hat{C}\left(u_{1}, \ldots, u_{d}\right)=\frac{1}{n} \sum_{k=1}^{n} 1\left(\hat{F}_{1}\left(x_{1}^{(k)}\right) \leq u_{1}, \ldots, \hat{F}_{d}\left(x_{d}^{(k)}\right) \leq u_{d}\right)
$$

can be used to guide the selection of the family of copulas $C\left(\cdot ; \theta_{0}\right)$ required by the above methods for fitting copulas to observations.

We conclude with the comment that alternatives to the copula-based representations of the multivariate distribution of $X$ in Eqs. 9 and 11 can be considered, e.g., families of parametric models $\{F(x ; \theta)\}$ indexed by $\theta$ for the distribution $F(x)$ of $X$ or empirical distributions $\hat{F}(x)$. The accuracy of the distributions $\hat{F}(x)$ depends on the sample size and 
the dimension of $X$. Let $X^{(k)}, k=1, \ldots, n$, be independent copies of a random vector $X$ with distribution $F$. The estimator,

$$
\hat{\bar{F}}(x)=1-\frac{1}{n} \sum_{k=1}^{n} 1\left(\cap_{i=1}^{d}\left\{X_{i}^{(k)} \leq x_{i}\right\}\right), \quad x=\left(x_{1}, \ldots, x_{d}\right),
$$

of $\bar{F}(x)=1-F(x)$ is unbias, i.e., $E[\hat{\bar{F}}(x)]=\bar{F}(x)$, with variance $\sigma(n)^{2}=F(x) \bar{F}(x) / n$ so that $P(|\hat{F}(x)-F(x)|>\varepsilon) \leq \sigma(n)^{2} / \varepsilon^{2}, \varepsilon>0$, by the Chebyshev inequality, which means that $\hat{\bar{F}}(x) \rightarrow \bar{F}(x)$ in probability as $n \rightarrow \infty[9]$ (Sects. 2.12-2.13). For large $n$, the estimator $\hat{\bar{F}}(x)$ is approximately Gaussian with mean $\bar{F}(x)$ and variance $\sigma(n)^{2}=F(x) \bar{F}(x) / n$ so that $(F(x)-\hat{F}(x)) / \sigma(n)$ takes values in $\left(-z_{\gamma / 2}, z_{\gamma / 2}\right)$ with probability $1-\gamma$. Accordingly, $\bar{F}(x)$ is in the range $\left(\hat{\bar{F}}(x)-\sigma(n) z_{\gamma / 2}, \hat{\bar{F}}(x)-\sigma(n) z_{\gamma / 2}\right)$ with probability $1-\gamma$, where $z_{\gamma / 2}=\Phi^{-1}(1-\gamma / 2)$. The absolute width $w(n):=2 \sigma(n) z_{\gamma / 2}$ of this confidence interval can be misleading when $\bar{F}(x)$ is very small, e.g., $w(n) \simeq 10^{-4} / \sqrt{n}$ for $\bar{F}(x)=10^{-8}$ which may suggest that $\hat{\bar{F}}(x)$ is accurate. The coefficient of variation $\sigma(n) / \bar{F}(x)$ of the estimator in Eq. 13 is approximately equal to $(\bar{F}(x) n)^{-1 / 2}$ for small probabilities $\bar{F}(x)$. It is of order $10^{2}$ for $\bar{F}(x)=10^{-8}$ and $n=10^{4}$.

The accuracy of $\hat{\bar{F}}(x)$ depends in a complex manner on the functional form of the right tail of $F$, the threshold $x$, the sample size $n$, and the dimension of $X$. We also note that the empirical distributions may yield accurate information on the dependence between the components of $X$ in the mid range of $F(x)$ for sufficiently large $n$ but provides no information

beyond data, i.e., $\hat{\bar{F}}$ cannot be used to characterize the tails of $F(x)$. Models of $F(x)$ are needed to extrapolate $\hat{\bar{F}}(x)$ beyond observations.

\subsection{Extreme value theory}

We have seen that independent observations of a random vector $X$ can be used to construct empirical distributions of this vector, i.e., samples of the estimator in Eq. 13, and that empirical distributions can be used to characterize random vectors provided they are based on sufficiently large sets of observations. Generally, the sample size is insufficient to obtain accurate empirical distributions. It is common to postulate parametric models for the copula or the joint distribution of $X$ and estimate their unspecified parameters from observations. The main advantage of the parametric models is that they can be fitted to relatively small sets of observations. The main limitations are that the dependence between the components of $X$ and the tails of the distribution of this vector can be severely constrained by the functional form of the postulated model. Whether parametric models for copulas are superior to those for joint distributions is questionable [15].

Our discussion is limited to two-dimensional random vectors $X$ whose components can be an intensity measure $X_{1}:=S_{a}(T)$ and a structural demand parameter $X_{2}:=D$. Our focus here is on the dependence between simultaneously large values of $X_{1}$ and $X_{2}$, rather than their overall dependence of these random variables. The analysis provides additional insight on the usefulness of $S_{a}(T)$ as a seismic intensity measure since it quantifies the dependence between simultaneously large IMs and structural responses. 
Since copulas are not ideal models for the dependence between components of random vectors, and particularly for large values of these components, we also explore the relationship between $X_{1}:=S_{a}(T)$ and $X_{2}:=D$ by using concepts of the multivariate extreme value theory (MEVT). If $S_{a}(T)$ and $D$ show significant dependence, then $S_{a}(T)$ is a satisfactory seismic intensity measure for ground motions with large IMs. Otherwise, $S_{a}(T)$ is an unsatisfactory IMs for large structural demand parameters $D$. The relationship between large demand parameters $D$ and $S_{a}(T)$ is particularly important since large demand parameters are likely to cause extensive damage or even structural failure.

The following sections present a heuristic review of concepts of the MEVT and computational tools relevant to our discussion. These concepts and tools are used in a subsequent section to quantify the dependence between simultaneously large samples of $S_{a}(T)$ and $D$ for several structural systems and ground acceleration processes. For a complete, rigorous discussion on the extreme value theory, see [18].

Let $\left\{x^{(k)}\right\}, k=1, \ldots, n$, be $n$ independent samples of a two-dimensional random vector $X=\left(X_{1}, X_{2}\right)$. Our objective is to quantify the dependence between simultaneously large values of $X_{1}$ and $X_{2}$. We first examine this dependence for the special case in which the components of $X$ are identically distributed. We then relax this constraint.

\subsubsection{Identically distributed components}

Suppose $X_{1}, X_{2}>0$ almost surely (a.s.), i.e., $P\left(X_{i}>0\right)=1$ for $i=1,2$, and the marginal distributions of $X=\left(X_{1}, X_{2}\right)$ coincide, i.e., $F_{1}=F_{2}$, so that the components of $X$ have similar scales. The polar representation of $X$ has the form

$$
X=\left(X_{1}, X_{2}\right)=(V \cos (\Theta), V \sin (\Theta)),
$$

where $V=\|X\|$ is a norm in $\mathbb{R}^{2}$ and $\Theta=\tan ^{-1}\left(X_{2} / X_{1}\right)$. The polar representation of the samples $\left\{x^{(k)}\right\}$ of $X$ is

$$
x^{(k)}=\left(x_{1}^{(k)}, x_{2}^{(k)}\right)=\left(v^{(k)} \cos \left(\theta^{(k)}\right), v^{(k)} \sin \left(\theta^{(k)}\right)\right), \quad k=1, \ldots, n,
$$

with the notations in Eq. 14. Set $v_{0}>0$ and relatively large. Samples of $X$ with distance to origin $v^{(k)}>v_{0}$ are of interest. The selection of $v_{0}$ is critical to assure that the right tails of the components of $X$ are accurately represented [17].

Let $\left\{y^{\left(k_{1}\right)}, \ldots, y^{\left(k_{m}\right)}\right\}$ denote the subset of $\left\{x^{(1)}, \ldots, x^{(n)}\right\}, m \leq n$, such that $v^{\left(k_{j}\right)}>v_{0}$, $j=1, \ldots, m$. The histogram $h(\theta)$ of $\left\{\theta^{\left(k_{1}\right)}, \ldots, \theta^{\left(k_{m}\right)}\right\}$ with support $[0, \pi / 2]$ is used to characterize the dependence between samples of $X$ with norm exceeding $v_{0}$. If most of the mass of $h(\theta)$ is concentrated at $\theta=0$ and $\pi / 2$, extremes of $X_{1}$ and $X_{2}$ are nearly independent. If most of the mass of $h(\theta)$ is concentrated at $\theta=\pi / 4$, extremes of $X_{1}$ and $X_{2}$ are strongly dependent. Histograms $h(\theta)$ between these limit cases describe various degrees of dependence between the components of large values of $\|X\|$.

Suppose $X_{i}=\lambda G_{0}^{2}+(1-\lambda) G_{i}^{2}, i=1,2$, where $G_{0}, G_{1}$, and $G_{2}$ are independent standard Gaussian variables. The components $X_{1}$ and $X_{2}$ of $X$ follow the same distribution. They are perfectly dependent for $\lambda=1$ and independent for $\lambda=0$. The left and right panels of Fig. 3 show scatter plots for samples of the random vector $Y=\left(Y_{1}, Y_{2}\right)$, i.e., samples of $X$ with distance to the origin of the coordinate system larger than $v_{0}$, and histograms of 

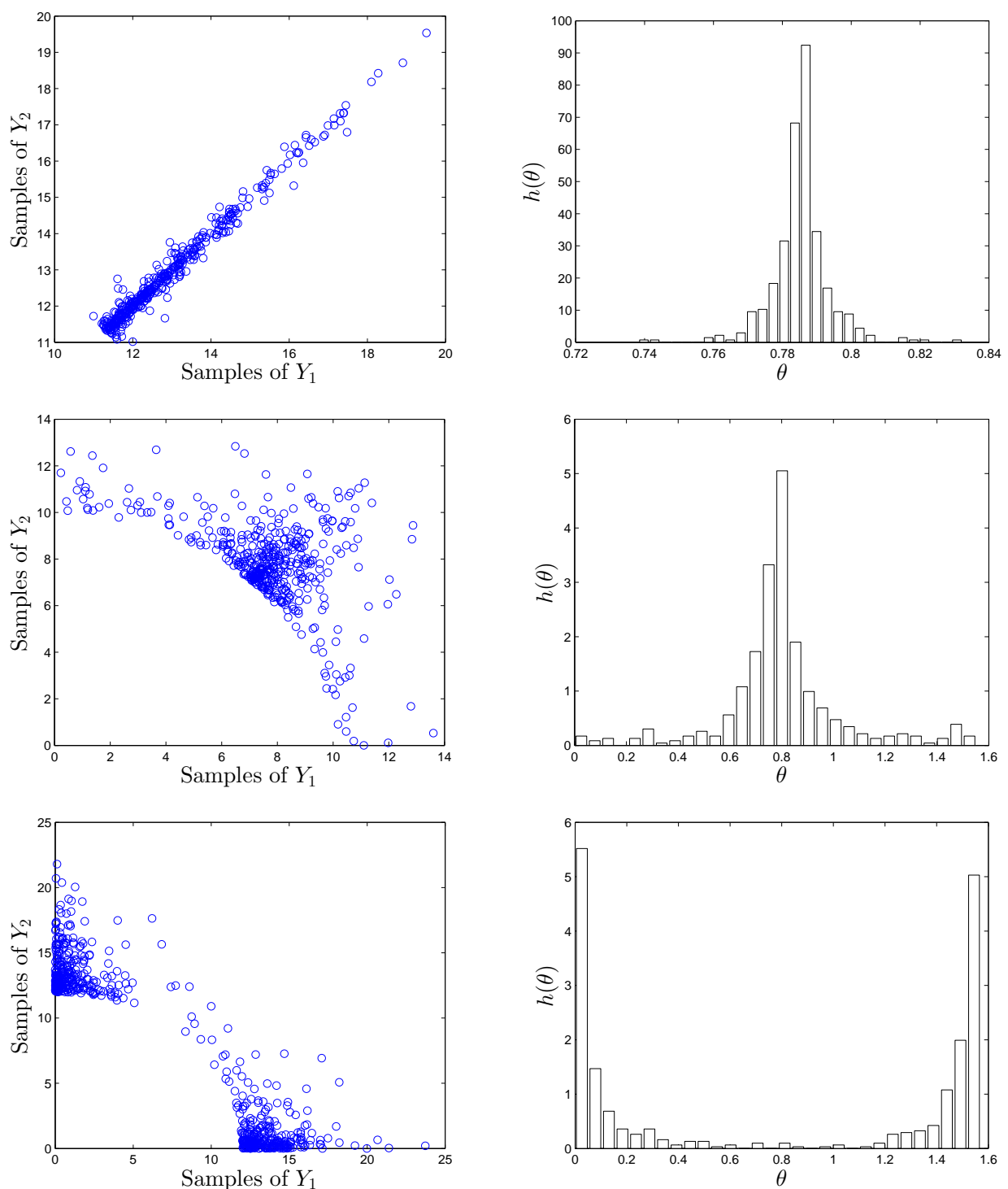

Figure 3: Large samples (left panel) and angular measures (right panel) for $\lambda=0.9$ and $v_{0}=16$ (top panels), $\lambda=0.5$ and $v_{0}=10$ (middle panels), and $\lambda=0.1$ and $v_{0}=12$ (bottom panels)

the angular measure $\Theta=\tan ^{-1}\left(Y_{2} / Y_{1}\right)$. The top, middle, and bottom panels correspond to $\lambda=0.9,0.5$, and 0.1 and thresholds $v_{0}=16,10$, and 12 . The resulting sample sizes of the vectors $Y$ are 435, 448, and 585, respectively, and have been extracted from $n=100,000$ independent samples of $X$. The components of $Y$ are strongly and weakly dependent for $\lambda=0.9$ and 0.1 . The angular measures $h(\theta)$ in the right panels quantifies the degree of dependence between the components of $Y$. For $\lambda=0.9$, the samples of $Y$ are aligned along the $45^{\circ}$ line and the angular measure is concentrated about $\pi / 4 \simeq 0.7854$. The support of $h(\theta)$ is approximately [0.74,0.83]. This shows that simultaneously large values of $X_{1}$ and $X_{2}$ are strongly dependent. For $\lambda=0.5$, the samples of $Y$ are less concentrated along the $45^{\circ}$ line and $h(\theta)$ takes non-zero values in $[0, \pi / 2]$. For $\lambda=0.1$, the samples of $Y$ cluster on 
the axis of the system of coordinates and most of the mass of $h(\theta)$ is concentrated is small vicinities of $\theta=0$ and $\pi / 2$. This means that large values of $X_{1}$ are likely to be associated with small values of $X_{2}$ and viceversa.

If the vector $\left(X_{1}, X_{2}\right)$ in Fig. 3 was $\left(S_{a}(T), D\right)$, we would have concluded that $S_{a}(T)$ is a very good, acceptable, and unacceptable measure of seismic intensity for $\lambda=0.9,0.5$, and 0.1 , respectively, since the uncertainty in the conditional random variable $D \mid S_{a}(T)$ is very small, moderate, and large for $\lambda=0.9,0.5$, and 0.1 . This means that the knowledge of $S_{a}(T)$ provides almost no information on $D$ for $\lambda=0.1$.

\subsubsection{General case}

The special case just considered is rarely encountered in applications. Usually, the components of $X$ have different scales and both the marginal distributions $\left\{F_{i}\right\}$ of the components $\left\{X_{i}\right\}$ of $X$ and the distribution $F$ of $X$ are not known.

Suppose first that the marginal distributions $\left\{F_{i}\right\}$ are known and that, as previously, $n$ independent samples $\left\{x^{(k)}\right\}, k=1, \ldots, n$, of $X$ are available. Denote by $Z$ a random vector obtained from $X$ by scaling its components such that they follow the same distribution, e.g., the unit Pareto distribution. Note that the components of $Z$ are identically distributed but are not independent. The previous approach can be used in the $z$-space to characterize the dependence between simultaneously large values of the components of $X$.

Generally, neither the distribution $F$ of $X$ nor the marginal distributions $\left\{F_{i}\right\}$ of this vector are known and the available samples $\left\{x^{(k)}\right\}, k=1, \ldots, n$, are insufficient to estimate these distributions accurately. We use an alternative approach, referred to as the ranks method, to estimate the dependence between large values of the components of $X$. The implementation of this method does not require knowledge of the distributions $F$ and $\left\{F_{i}\right\}$ [18]. The output of the ranks method is an estimate of the angular or spectral measure $s(\theta)$ which, for $d=2$, is analogue of the histogram $h(\theta)$ introduced earlier in this section. We use the angular measure to assess the dependence between the components of the twodimensional random vector $X=\left(X_{1}=S_{a}(T), X_{2}=D\right)$.

The implementation of the ranks method involves the following three steps [18] (Sect. 9.2). First, map the observations $\left\{x^{(k)}\right\}$ into ranks $\left\{r_{i}^{(k)}\right\}, i=1,2$, defined by

$$
r_{i}^{(k)}=\sum_{l=1}^{n} 1\left(x_{i}^{(l)} \geq x_{i}^{(k)}\right), \quad k=1, \ldots, n .
$$

Note that the ranks of the largest and smallest samples of $X_{i}$ are 1 and $n$, i.e., the opposite of the rank in Eq. 10. Second, represent the vectors $\left\{\left(n_{0} / r_{1}^{(k)}, n_{0} / r_{2}^{(k)}\right)\right\}$ in polar coordinates, i.e.,

$$
\left(n_{0} / r_{1}^{(k)}, n_{0} / r_{2}^{(k)}\right)=\left(\rho^{(k)} \cos \left(\alpha^{(k)}\right), \rho^{(k)} \sin \left(\alpha^{(k)}\right)\right), \quad k=1, \ldots, n,
$$

where $0<n_{0} \ll n$ is an integer and $\left(\rho^{(k)}, \alpha^{(k)}\right)$ is defined as $(V, \Theta)$ in Eq. 14. Note that the length $\rho^{(k)}=\left\|x^{(k)}\right\|$ of the position vector of $x^{(k)}$ increases with its distance to the origin of the system of coordinates. Third, construct the histogram of the directions $\left\{\alpha^{(k)}, \rho^{(k)}>1\right\}$ whose support is $[0, \pi / 2]$. The normalized version of this histogram is an estimate of the angular measure $s(\theta)$ [18] (Chap. 9). The selection of $n_{0}$ is very important. If $n_{0}$ is too small, the statistical uncertainty in the estimates of $s(\theta)$ can be significant. If $n_{0}$ is too large, 
tail properties can be misrepresented since many of the retained data are in the body of the distribution of $X$.

The $n=100,000$ samples of the random vector $X=\left(X_{1}, X_{2}\right)$ used to construct the plots in Fig. 3 are also used for the plots in Fig. 4. The left and right panels show the samples
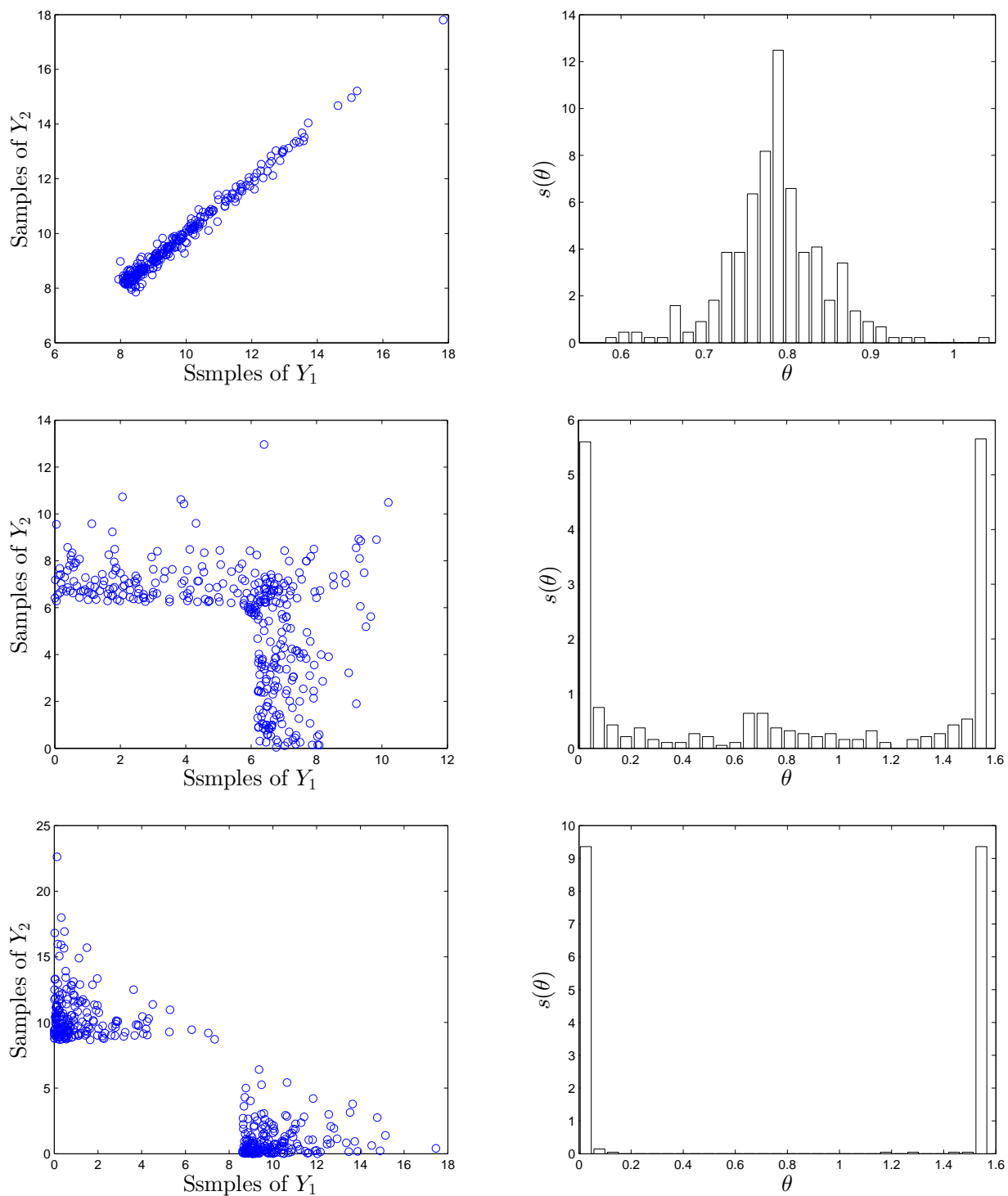

Figure 4: Large samples (left panel) and angular measures (right panel) for $\lambda=0.9$ (top panels), $\lambda=0.5$ (middle panels), and $\lambda=0.1$ (bottom panels)

$\left\{\left(n_{0} / r_{1}^{(k)}, n_{0} / r_{2}^{(k)}\right)\right\}$ retained to construct estimates of the angular measure $s(\theta)$. The top, middle, and bottom plots are for $\lambda=0.9,0.5$, and 0.1 and use the top $n_{0}=200$ samples of $X$. The histograms of the angular measures in Figs. 3 and 4 are similar for $\lambda=0.1$ and 0.9 . They capture accurately the strong and the weak dependence between large values of $X_{1}$ and $X_{2}$, although their construction uses rather different concepts. The angular measures in Figs. 3 and 4 are less similar for $\lambda=0.5$. The histogram $h(\theta)$ in Fig. 3 based on heuristic considerations suggests a stronger dependence between $X_{1}$ and $X_{2}$ than that quantified by 
the angular measure $s(\theta)$ in Fig. 4. Nevertheless, the histograms $h(\theta)$ and the angular measures $s(\theta)$ show consistently that the dependence between large values of $X_{1}$ and $X_{2}$ for $\lambda=0.5$ is weaker and stronger than that for $\lambda=0.9$ and $\lambda=0.1$.

\section{Dependence of $D$ and $S_{a}(T)$}

The dependence between demand parameters $D$ and intensity measures $S_{a}(T)$ is calculated for a Bouc-Wen SDOF system (the following two subsections) and a linear multi-degree of freedom (MDOF) system (the last subsection). The seismic acceleration $A(t)$ is a stationary Gaussian process with specified spectral density. The metrics in the previous section are used to quantify the dependence between $D$ and $S_{a}(T)$ and assess the potential of vectorvalued IMs consisting of multiple ordinates of $S_{a}(T)$.

\subsection{Copula models}

Suppose $X(t)$ is the displacement of a Bouc-Wen SDOF system defined by

$$
\begin{gathered}
\ddot{X}(t)+2 \zeta \nu_{0} \dot{X}(t)+\nu_{0}^{2}(\rho X(t)+(1-\rho) W(t))=-A(t), \quad \text { where } \\
\dot{W}(t)=\gamma \dot{X}(t)-\alpha|\dot{X}(t)||W(t)|^{\chi^{-1}} W(t)-\beta \dot{X}(t)|W(t)|^{\chi}
\end{gathered}
$$

$\nu_{0}$ are $\zeta$ as in Eq. 1, $\alpha, \beta, \gamma, \rho$, and $\chi$ are positive constants, and $A(t)$ denotes the seismic acceleration process. The parameters $\alpha, \beta, \gamma, \rho$, and $\chi$ control the model behavior, e.g., the system is linear for $\rho=1$ and, for $\rho \neq 1$, its nonlinear/hyteretic behavior depends strongly on $\beta$ and $\gamma$.


Figure 5: Spectral density of $A(t)$ and $n=500$ independent samples of $S_{a}(T)$ and $D$ (left and right panels)

The numerical results in this and the subsequent section are for $\nu_{0}=2 \pi, \zeta=0.05$, $\alpha=0.5, \beta=5, \gamma=3, \rho=0.1$, and $\chi=1$. The ground acceleration $A(t)$ is given by the specific barrier model (SBM) for a seismic event with magnitude $m=5.0$, source-to-site distance $r=185 \mathrm{~km}$, and a rock site. It is a zero-mean stationary Gaussian process with spectral density in the left panel of Fig. 5. The right panel of the figure shows $n=500$ independent samples of $\left(X_{1}:=S_{a}(T), X_{2}=D\right)$ for $\tau=20$ seconds. The visual inspection 
of this scatter plot suggests that $S_{a}(T)$ and $D$ are weakly dependent so that $S_{a}(T)$ is an unsatisfactory IM for Bouk-Wen oscillators. This observation is at variance with our finding for the Duffing oscillator in Fig. 1. It also suggests that $S_{a}(T)$ provides satisfactory IMs only for SDOF systems whose displacements are perturbations of displacements of linear SDOF used to construct $S_{a}(T)$.

Our objective is to quantify the relationship between the components $X_{1}=S_{a}(T)$ and $X_{2}=D$ of $X$ based on finer tools than correlation coefficients. To achieve this objective, we employ the copula and extreme value technologies in this and the subsequent section.

Let $\left\{x^{(k)}\right\}, k=1, \ldots, n$, be $n=500$ independent samples of $X$. These samples are shown in the right panel of Fig. 5. The left and right panels in Fig. 6 show empirical marginal distributions of the components of $X$ corresponding to these samples of $X$. The empirical copula in the left panel of Fig. 7 has been calculated from Eq. 12 by using observation ranks.


Figure 6: Empirical distributions of $X_{1}=S_{a}(T)$ and $X_{2}=D$ (left and right panels) and empirical copula $\hat{C}(u, v)$ (right panel)

The right panel in this figure is the Clayton copula fitted to data, an Archimedean copula
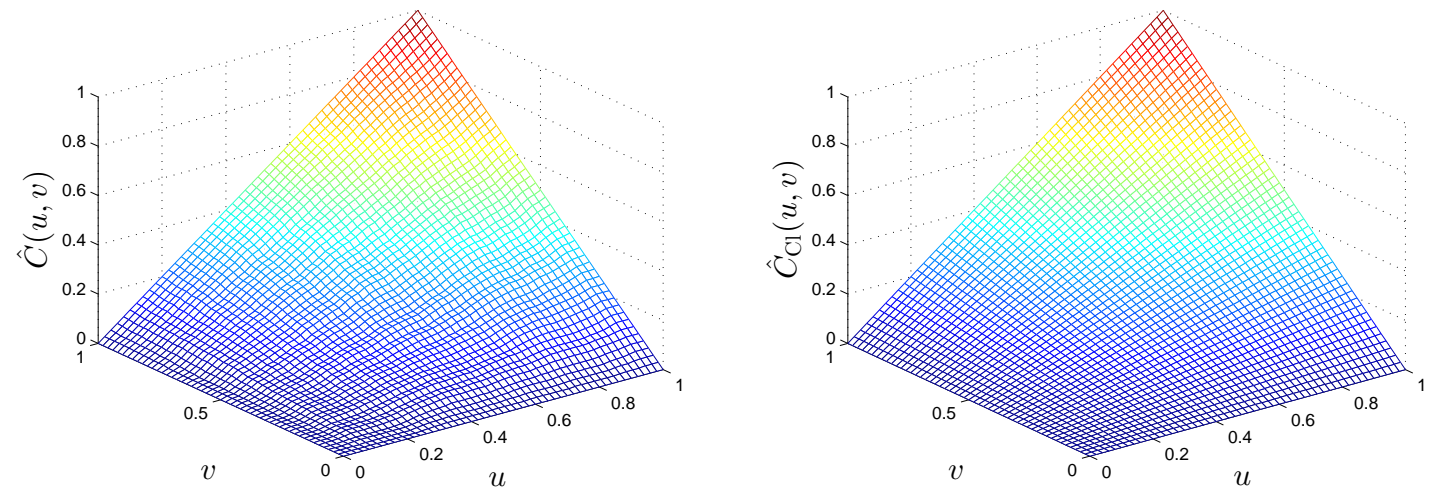

Figure 7: Empirical and fitted Clayton copulas (left and right panels)

defined by $C(u, v)=\varphi^{-1}(\varphi(u)+\varphi(v))$ with $\varphi(t)=\left(t^{-\theta}-1\right) / \theta$ so that it has the expression $C(u, v)=\left(u^{-\theta}+v^{-\theta}-1\right)^{-1 / \theta}$, see Eq. 7. The MATLAB function copulafit.m was employed to find the MLE $\hat{\theta}=1.4509 \times 10^{-6}$ of $\theta$. Accordingly, the random variables $X_{1}=S_{a}(T)$ and 
$X_{2}=D$ are nearly independent (see comments following Eq. 7). The left and right panels of Fig. 8 show differences between the empirical copula $\hat{C}(u, v)$ and the fitted $\hat{C}_{\mathrm{CL}}(u, v)$


Figure 8: Difference $\hat{C}(u, v)-\hat{C}_{\mathrm{CL}}(u, v)$ between empirical and fitted Clayton copulas (left panel) and contours of $\hat{C}(u, v)$ and $\hat{C}_{\mathrm{CL}}(u, v)$ (right panels)

and contour lines of the empirical and fitted Clayton copulas in solid and dash lines. The differences are minor and the contour lines are similar.

Estimates $\hat{F}\left(x_{1}, x_{2}\right)$ of the joint distribution $F\left(x_{1}, x_{2}\right)$ of $X$ can be obtained from copulas, e.g., the empirical or fitted Clayton copula in Fig. 7, the empirical distributions in Fig. 6, and Eq. 11. For example, the estimate of $F\left(x_{1}, x_{2}\right)$ based on the Clayton copula has the form

$$
\hat{F}_{\mathrm{Cl}}\left(x_{1}, x_{2}\right)=\left(\hat{F}_{1}\left(x_{1}\right)^{-\hat{\theta}}+\hat{F}_{2}\left(x_{2}\right)^{-\hat{\theta}}-1\right)^{-1 / \hat{\theta}} .
$$

Alternatively, observations can be used directly to calculate the estimate $\hat{F}\left(x_{1}, x_{2}\right)$ of $F\left(x_{1}, x_{2}\right)$ in Eq. 13 with $d=2$.

The left and right panels of Fig. 9 show the empirical joint distribution $\hat{F}\left(x_{1}, x_{2}\right)$ of
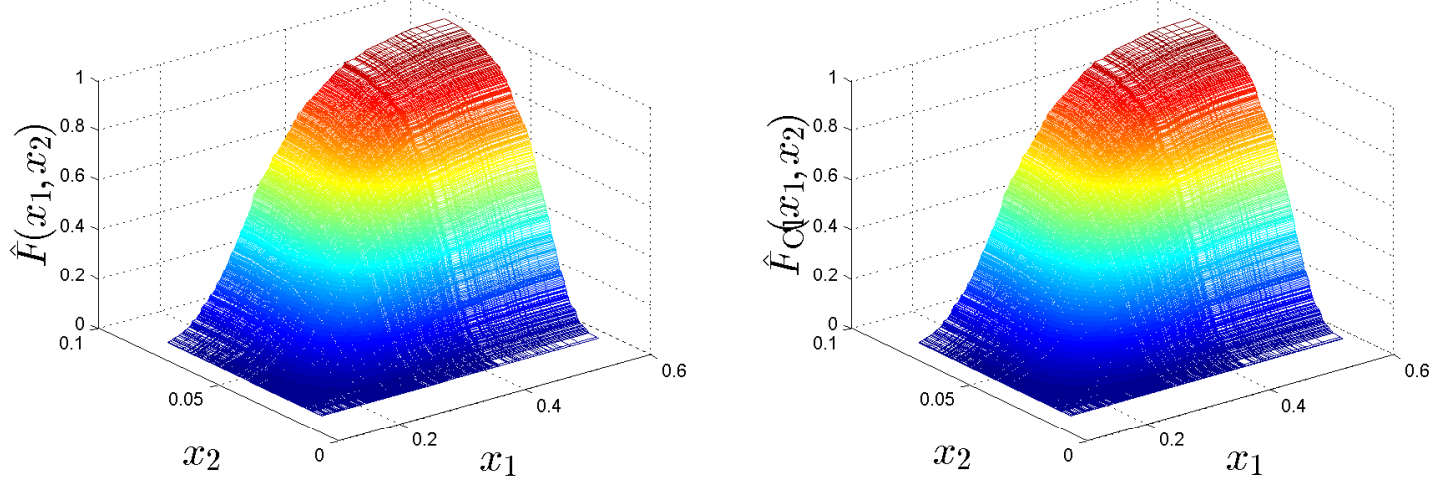

Figure 9: Empirical $\hat{F}\left(x_{1}, x_{2}\right)$ and fitted Clayton $\hat{F}_{\mathrm{Cl}}\left(x_{1}, x_{2}\right)$ distributions (left and right panels)

$X$ and the joint distribution $\hat{F}_{\mathrm{Cl}}\left(x_{1}, x_{2}\right)$ of this random vector given by Eq. 19. Differences 

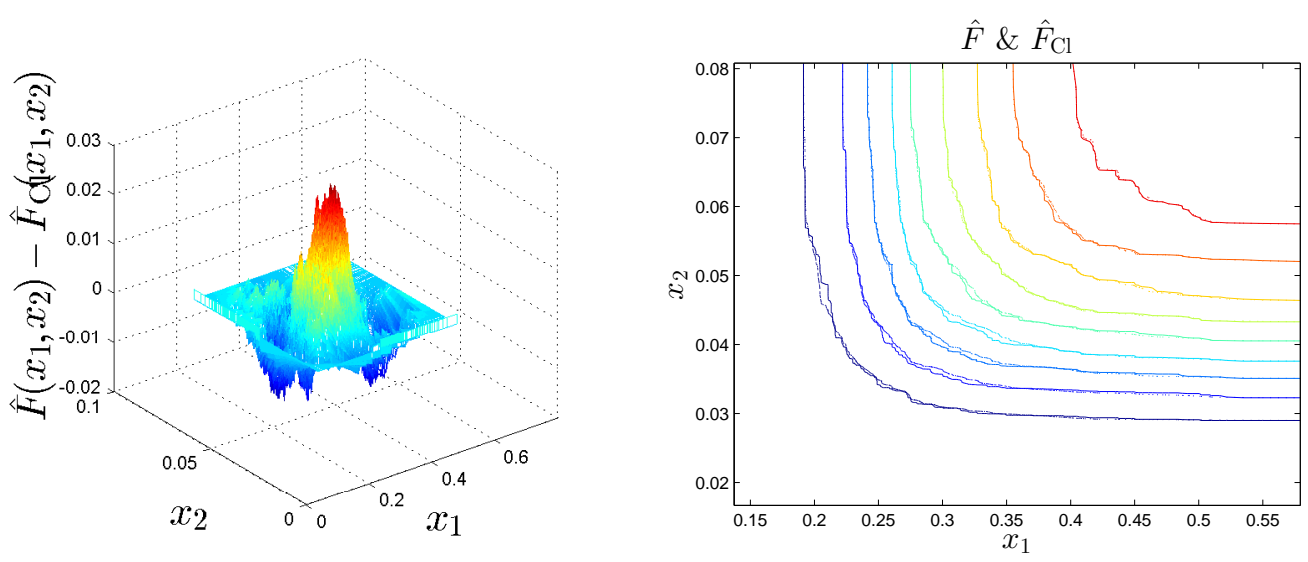

Figure 10: Difference $\hat{F}\left(x_{1}, x_{2}\right)-\hat{F}_{\mathrm{Cl}}\left(x_{1}, x_{2}\right)$ between empirical and fitted Clayton distributions (left panel) and contours of $\hat{F}$ and $\hat{F}_{\mathrm{Cl}}$

between the distributions $\hat{F}\left(x_{1}, x_{2}\right)$ and $\hat{F}_{\mathrm{Cl}}\left(x_{1}, x_{2}\right)$ are shown in the left panel of Fig. 10 . Contour lines of $\hat{F}$ and $\hat{F}_{\mathrm{Cl}}$ are in the right panel of this figure. The contour lines of the distribution $\hat{F}_{1}\left(x_{1}\right) \hat{F}_{2}\left(x_{2}\right)$ are indistinguishable at the figure scale from those of the empirical joint distribution $\hat{F}\left(x_{1}, x_{2}\right)$, where $\hat{F}_{k}\left(x_{k}\right), k=1,2$, are the empirical distributions in Fig. 6 . This indicates, in agrement with our previous comments, that $X_{1}=S_{a}(T)$ and $X_{2}=D$ are nearly independent so that knowledge of $X_{1}=S_{a}(T)$ does not reduce the uncertainty in $X_{2}=D$. This means that $S_{a}(T)$ is an unsatisfactory IM for this structural system.

\subsection{Extreme value models}

Let $X=\left(X_{1}=S_{a}(T), X_{2}=D\right)$ be the two-dimensional random vector in the previous subsection with samples $\left\{x^{(k)}\right\}, k=1, \ldots, n$. It was found that the components of $X$ are nearly independent. We further examine the dependence between the components of $X$ by focusing on simultaneously large values of $S_{a}(T)$ and $D$. Estimates of the angular measure $s(\theta)$ defined in Sect. 3.3.2 are used to quantify this dependence.

The samples in the left panel in Fig. 11 are those in the left panel of Fig. 5. The samples marked with circles denote the top $n_{0}=20$ samples of $\left(S_{a}(T), D\right)$ used to estimate the angular measure $s(\theta)$ shown in the right panel of the figure. Since most of the mass of the estimate of $s(\theta)$ is concentrated in small vicinities of $\theta=0$ and $\theta=\pi / 2$, simultaneously large values of $S_{a}(T)$ and $D$ are unlikely. Hence, large values of $D$ are associated with small, moderate, and large values of $S_{a}(T)$, which implies that $S_{a}(T)$ is an unsatisfactory IM.

In summary, Sect. 4.1 shows that the overall dependence between $S_{a}(T)$ and $D$ is weak. This section shows that the dependence between simultaneously large values of $S_{a}(T)$ and $D$ is also weak. This means that the conditional random variable $D \mid S_{a}(T)$ and the random variable $D$ have similar distributions so that $P\left(D \in I \mid S_{a}(T)\right) \simeq P(D \in I)$, i.e., the fragility of the Bouc-Wen system under consideration is nearly independent of $S_{a}(T)$. We conclude that $S_{a}(T)$ provides limited information on $D$ for Bouc-Wen SDOF systems so that fragilities for these systems defined as functions of $S_{a}(T)$ are unsatisfactory. This observation is of significant concern since the Bouc-Wen SDOF system is a simplistic model 



Figure 11: Samples of $\left(X_{1}:=S_{a}(T), X_{2}=D\right)$ and estimates of the angular measure $s(\theta)$ (left and right panels)

for realistic structures, large values of the demand parameter $D$ and the intensity measure $S_{a}(T)$ are nearly independent, and large values of $D$ are associated with excessive damage or even structural collapse.

\subsection{Vector-valued intensity measures}

To improve the explanatory power of scalar intensity measures, it is has been proposed to characterize seismic ground acceleration by vector-valued IMs, e.g., values of $S_{a}(T)$ at two or more selected periods [2]. While vector-valued IMs constitute an improvement over their scalar-valued versions, they do not address the fundamental weakness of this class of IMs, i.e., the fact that responses of complex, nonlinear structures and responses of selected SDOF linear oscillators to seismic ground accelerations can differ significantly and, generally, are weakly dependent. This suggests that the uncertainty in the responses of realistic structures cannot be reduced to an acceptable level by conditioning on multiple ordinates of $S_{a}(T)$.

The following examples examines the dependence between distinct ordinates of $S_{a}(T)$ and between demand parameters for two-degree of freedom linear systems and ordinates of $S_{a}(T)$ at the system modal periods and the dependence between simultaneously large values of ordinates of $S_{a}(T)$ and $D$.

\subsubsection{Ordinates of $S_{a}(T)$}

Consider two SDOFs with parameters $\left(\zeta, T_{1}\right)$ and $\left(\zeta, T_{2}\right)$ subjected to a band limited Gaussian white noise process with mean zero, one-sided spectral density $g(\nu)=1(0 \leq \nu \leq$ $\bar{\nu}) / \bar{\nu}$, and cutoff frequency $\bar{\nu} \gg \max \left\{\nu_{1}, \nu_{2}\right\}$, where $\nu_{k}=2 \pi / T_{k}, k=1,2$. In the stationary regime the displacements $X_{k}(t), k=1,2$, of the oscillators are stationary Gaussian processes with mean zero and spectral densities $g_{k}(\nu)=1(0 \leq \nu \leq \bar{\nu})\left|h_{k}(\nu)\right|^{2} / \bar{\nu}$, where $h_{k}(\nu)$ denotes the frequency response function for the $\left(\zeta, T_{k}\right)$-oscillator [19] (Sect. 5.2.2).

The correlation coefficient between the random variables $X_{1}(t)$ and $X_{2}(t)$ at an arbitrary time $t$ has the expression

$$
\rho_{X_{1}(t), X_{2}(t)}=8 \lambda^{3 / 2}(1+\lambda) \zeta^{2} /\left[4 \zeta^{2} \lambda(1+\lambda)^{2}-\left(1-\lambda^{2}\right)^{2}\right]
$$


where $\lambda=\nu_{1} / \nu_{2}<1$ [7]. Figure 12 shows the correlation coefficients $\rho_{X_{1}(t), X_{2}(t)}$ for $\zeta$

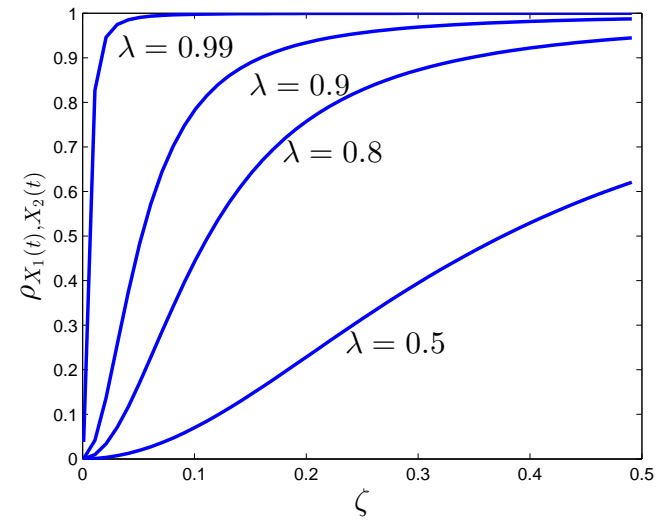

Figure 12: Correlation coefficients between $X_{1}(t)$ and $X_{2}(t)$ for several modal frequency ratios $\lambda=\nu_{1} / \nu_{2}$ and damping ratios $\zeta$

in the range $[0.001,0.5]$ and $\lambda=\nu_{1} / \nu_{2}=0.99,0.9,0.8$, and 0.5. Unless the frequencies $\left\{\nu_{k}\right\}$ are closely-spaced and/or damping ratios are large, the random variables $X_{1}(t)$ and $X_{2}(t)$ are weakly correlated and so are maxima of the processes $\left|X_{1}(t)\right|$ and $\left|X_{2}(t)\right|$, i.e., scaled versions of the ordinates of $S_{a}(T)$ for $T=T_{k}, k=1,2$. This suggests that vectorvalued IMs $\left\{S_{a}\left(T_{k}\right)\right\}, k=1,2, \ldots$, may be useful and may not justified for correlations $\rho_{X_{1}(t), X_{2}(t)} \simeq 0$ and $\rho_{X_{1}(t), X_{2}(t)} \simeq 1$ since the random variables $\left\{S_{a}\left(T_{k}\right)\right\}$ carry different and similar information, respectively. It is shown later in this section that vector-valued IMs only provide an incremental improvement over scalar-valued IMs since both types of IMs are based on the assumption that responses of complex nonlinear structural systems can be predicted from those of linear SDOFs with satisfactory accuracy.

The weak correlation between the processes $\left\{X_{k}(t)\right\}$ relates to the fact that most of their energy is concentrated in non-overlapping frequency bands centered on $\left\{\nu_{k}\right\}$ with widths depending on $\zeta$. Consider the special case of two weakly stationary processes $X_{k}(t), k=$ 1,2 , whose spectral densities $g_{k}(\nu)$ share no frequencies, i.e., $I_{k} \cap I_{l}=\emptyset, k \neq l$, with the notation $I_{k}=\left\{\nu \geq 0: g_{k}(\nu)>0\right\}$. Their spectral representations have the forms $X_{k}(t)=\int_{I_{k}} e^{i \nu t} d Z_{k}(\nu)$, where $Z_{k}(\nu)$ are uncorrelated processes with orthogonal increments such that $E\left[d Z_{k}(\nu) d Z_{k}(\eta)\right]=g_{k}(\nu) \delta(\nu-\eta) d \nu$ and $E\left[d Z_{1}(\nu) d Z_{2}(\eta)\right]=0$. We have

$E\left[X_{1}(t) X_{2}(s)\right]=E\left[\int_{I_{1}} e^{i \nu t} d Z_{1}(\nu)\left(\int_{I_{2}} e^{i \eta t} d Z_{2}(\eta)\right)^{*}\right]=\int_{I_{1} \times I_{2}} e^{i(\nu t-\eta s)} E\left[d Z_{1}(\nu) d Z_{2}(\eta)\right]$

which is zero since $E\left[d Z_{1}(\nu) d Z_{2}(\eta)\right]=0$.

\subsubsection{MDOF linear system}

Consider a linear structural system with $d>1$ degrees of freedom, modal frequencies $\left\{\nu_{k}\right\}$, modal damping ratios $\left\{\zeta_{k}\right\}$, modal shapes $\phi_{k}$, and participation factors $\left\{\Gamma_{k}\right\}$, $k=1, \ldots, d$. The system is subjected to a ground acceleration process $A(t)$ so that its 
displacement vector $X(t)$ has the form $X(t)=\sum_{k=1}^{d} \Gamma_{k} \phi_{k} Y_{k}(t) \in \mathbb{R}^{d}$, where the stochastic processes $Y_{k}(t)$ satisfy the equations

$$
\ddot{Y}_{k}(t)+2 \zeta_{k} \nu_{k} \dot{Y}_{k}(t)+\nu_{k}^{2} Y_{k}(t)=-A(t), \quad k=1, \ldots, d .
$$

Let

$$
Y(t)=c X(t)=\sum_{k=1}^{d} \Gamma_{k} c \Phi_{k} Y_{k}(t)=\sum_{k=1}^{d} \alpha_{k} Y_{k}(t)
$$

be a scalar response of interest, where $c$ is an $(1, d)$-matrix and $\alpha_{k}=\Gamma_{k} c \phi_{k} \in \mathbb{R}$. For example, $Y(t)$ with $c(1)=-1, c(2)=1$, and $c(i)=0, i \geq 2$, is the inter-story displacement between the second and the first floors.

Let $R_{k}=\max _{0 \leq t \leq \tau}\left|Y_{k}(t)\right|, k=1, \ldots, d$, and $R=\max _{0 \leq t \leq \tau}|Y(t)|$ denote the maxima of modal and system responses, where $\tau>0$ denotes the duration of the seismic input. We note that (1) the response $R$ is a possible structural demand parameter $D,(2)$ the stochastic processes $\left\{Y_{k}(t)\right\}$ and $Y(t)$ are dependent as functionals of the seismic acceleration process $A(t)$, so that the response maxima $\left\{R_{k}\right\}$ and $R$ are dependent random variables, (3) the system response $R$ cannot be obtained from $\left\{R_{k}\right\}$ since the maxima of $\left\{\left|Y_{k}(t)\right|\right\}$ occur at different times; it can bounded by

$$
R=\max _{0 \leq t \leq \tau}\left|\sum_{k=1}^{d} \alpha_{k} Y_{k}(t)\right| \leq \sum_{k=1}^{d}\left|\alpha_{k}\right| R_{k}
$$

and (4) $\left\{S_{a}\left(T_{k}\right)=\nu_{k}^{2} R_{k}\right\}$ are IMs corresponding to the periods $\left\{T_{k}=2 \pi / \nu_{k}\right\}$.

Suppose $A(t)$ is a stationary Gaussian band limited white noise (BLWN) process with mean 0 , variance 1 , and one-sided spectral density $g(\nu)=(1 / \bar{\nu}) 1(0 \leq \nu \leq \bar{\nu}), \bar{\nu}>\max _{k}\left\{\nu_{k}\right\}$. The stationary modal responses $\left\{Y_{k}(t)\right\}$ are Gaussian processes with mean 0 and spectral densities $\left\{g_{k}(\nu)=\left(\left|h_{k}(\nu)\right|^{2} / \bar{\nu}\right) 1(0 \leq \nu \leq \bar{\nu})\right\}$, where $h_{k}(\nu)$ denotes the frequency response function of a linear oscillator with damping ratio $\zeta_{k}$ and natural frequency $\nu_{k}$ [19] (Sect. 5.2.2). Our objective is to assess the relationships between $R$ and single and multiple ordinates of $S_{a}(T)$, i.e., single and multiple modal response maxima $\left\{R_{k}\right\}$.

The left and right panels in Fig. 13 show 10,000 independent samples of $\left(R_{1}, R_{2}\right),\left(R_{1}, R\right)$ and $\left(R_{2}, R\right)$ and estimates of the angular measures of these pairs of random variables. The plots are for $d=2, \nu_{1}=\pi, \nu_{2}=2 \pi, \zeta_{1}=\zeta_{2}=0.05, \bar{\nu}=20, \alpha_{1}=\alpha=0.3$, and $\alpha_{2}=1-\alpha$. The estimates of the angular measures use the top $n_{0}=100$ samples of $R$. The samples of $R, R_{1}$, and $R_{2}$ marked with circles indicate the subsets of data used to estimate the angular measures shown in the right panels of the figure. The visual inspection of the scatter plots in the left panels of the figure suggests that $\left(R_{1}, R_{2}\right),\left(R_{1}, R\right)$ and $\left(R_{2}, R\right)$ are weakly correlated and that the correlation between $\left(R_{1}, R\right)$ and $\left(R_{2}, R\right)$ is stronger that between $\left(R_{1}, R_{2}\right)$. The estimates of the correlation coefficients of $\left(R_{1}, R_{2}\right),\left(R_{1}, R\right)$ and $\left(R_{2}, R\right)$ based on the available samples are $0.0537,0.6356$, and 0.4520 . This show that $S_{a}\left(T_{1}\right)$ and $S_{a}\left(T_{2}\right)$ are weakly correlated, in agreement results in Fig. 12. The dependence of $\left(R_{1}, R\right)$ and $\left(R_{2}, R\right)$ is stronger than that of $\left(R_{1}, R_{2}\right)$ since $Y(t)$ is a linear form of $Y_{1}(t)$ and $Y_{2}(t)$. If a mode $k$ is dominant, then the dependence between $R$ and $R_{k}$ is much stronger than that between $R$ and $R_{l}, l \neq k$. If both modes contribute to structural response, e.g., $\alpha=0.3$ as in Fig. 13, then the correlations of $\left(R_{1}, R\right)$ and $\left(R_{2}, R\right)$ are similar and stronger than that of $\left(R_{1}, R_{2}\right)$ 

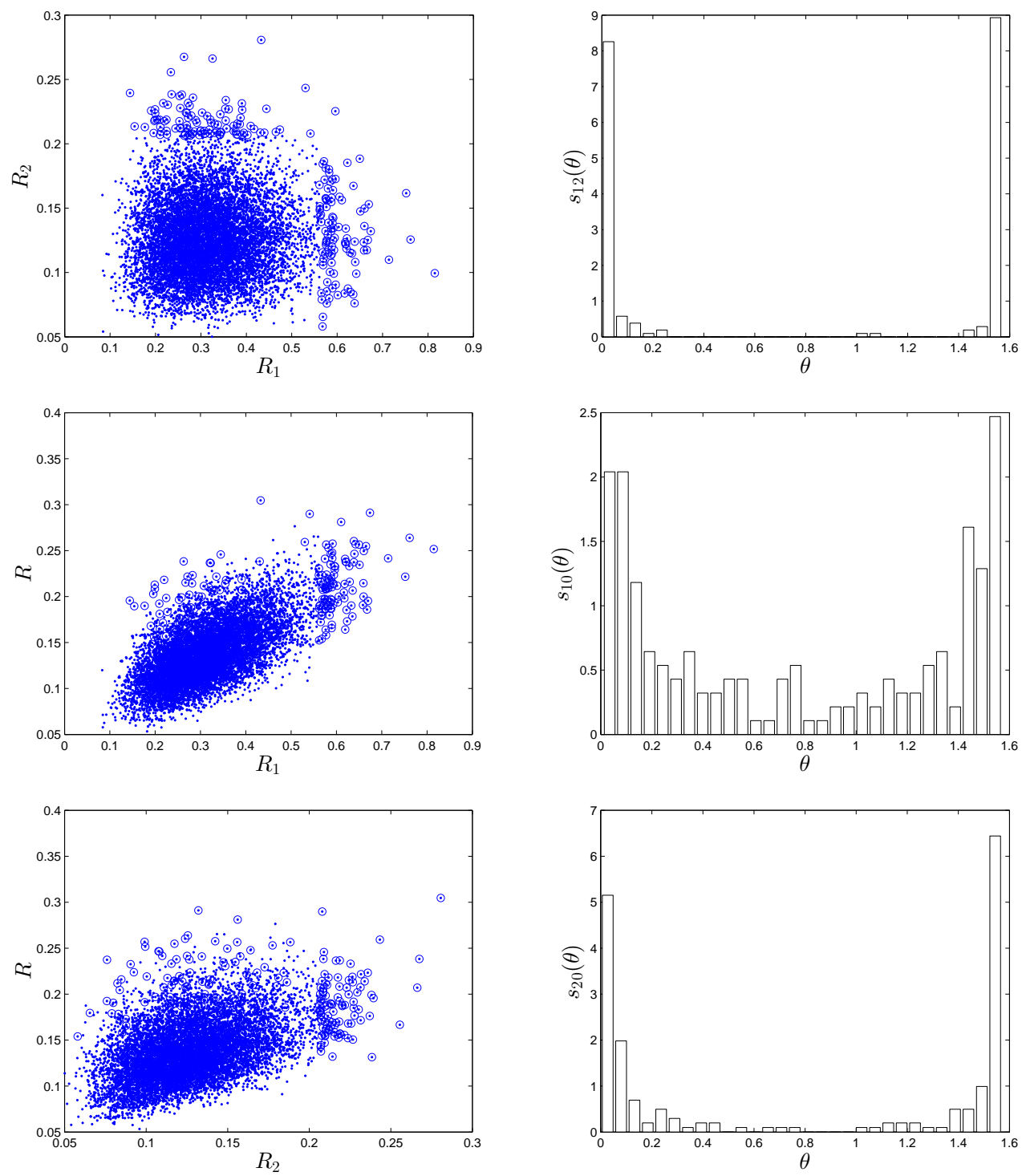

Figure 13: Samples of $\left(R_{1}, R_{2}\right),\left(R_{1}, R\right)$, and $\left(R_{2}, R\right)$ (left panels) and angular measures $s_{12}(\theta), s_{10}(\theta)$, and $s_{20}(\theta)$ of $\left(R_{1}, R_{2}\right),\left(R_{1}, R\right)$, and $\left(R_{2}, R\right)$ (right panels) for $\alpha=0.3$ and a BLWN $A(t)$

but remain relatively weak. This means that, although the vector-valued IM $\left(R_{1}, R_{2}\right)$ is superior to the single-valued IMs $R_{k}$, the conditional variable $R \mid\left(R_{1}, R_{2}\right)$ still has a rather large variance so that fragilities plotted against $\left(R_{1}, R_{2}\right)$ may not be satisfactory.

Following are three comments on the relationship between large values of ordinates of $S_{a}(T)$ and $R$. First, the setting in this example cannot be more favorable for the vectorvalued intensity measure $\left(S_{a}\left(T_{1}\right), S_{a}\left(T_{2}\right)\right)$. The structural system is linear with two degrees of freedom, the periods $T_{k}=2 \pi / \nu_{k}, k=1,2$, are the modal periods, and both modes contribute to the overall structural response. Second, the scatter plots in the left middle and bottom panels of Fig. 13 illustrate the variability of $R$ with $R_{1}$ and $R_{2}$, which suggests that even in this favorable situation the IM $\left(R_{1}, R_{2}\right)$ is not be satisfactory. Third, simultaneous 
large values of $R_{1}, R_{2}$, and $R$ are unlikely since the mass of the estimates of the angular measures $s_{12}(\theta), s_{10}(\theta)$, and $s_{20}(\theta)$ in the right panels of Fig. 13 is concentrated in small vicinities of $\theta=0$ and $\theta=\pi / 2$. The dependence between simultaneously large values of $R$ and $R_{1}$ is stronger that that between $R$ and $R_{2}$ since mode 1 contributes more than mode 2 to the response of interest. The near independence between simultaneously large values of $R_{1}$ and $R_{2}$ suggests that the angular measure of the random vector $\left(R_{1}, R_{2}, R\right)$ is concentrated in the planes $R_{1}=0$ and $R_{2}=0$ with properties similar to the angular measures shown in the middle and bottom right panels of Fig. 13. While the $\left(R_{1}, R_{2}\right)$-IM, or equivalently the $\left(S_{a}\left(T_{1}\right), S_{a}\left(T_{2}\right)\right)$-IM, is superior to the scalar-valued measures $R_{k}$, or equivalently $S_{a}\left(T_{k}\right)$, it is still an unsatisfactory IM because of the weak dependence between $R$ and $\left(R_{1}, R_{2}\right)$.

To further explore the relationship between the random variables $R, R_{1}$, and $R_{2}$, we estimate the correlation between large values of these variables and calculate properties of the conditional variables $R \mid\left(R_{1}, R_{2}\right)$ and $R \mid R_{k}$ under the assumption that $\left(R, R_{1}, R_{2}\right)$ is a Gaussian vector.

Let $\left(r^{(1)} \geq r^{(2)} \geq \cdots\right)$ denote the samples $\left\{r_{i}\right\}$ of $R$ for $\alpha=0.3$ set in decreasing order from the largest to the smallest and let $\left\{r_{k}^{(i)}\right\}, i=1,2, \ldots$, be the samples of $R_{k}, k=1,2$, corresponding to the samples $\left\{r^{(i)}\right\}$, i.e., the pairs $\left\{\left(r_{1}^{(1)}, r_{2}^{(1)}\right),\left(r_{1}^{(2)}, r_{2}^{(2)}\right), \ldots\right\}$ correspond to $\left\{r^{(1)}, r^{(2)}, \ldots\right\}$. Denote by $\hat{\rho}_{m, 10}, \hat{\rho}_{m, 20}$, and $\hat{\rho}_{m, 12}$ estimates of the correlation coefficients of $\left(R_{1}, R\right),\left(R_{2}, R\right)$, and $\left(R_{1}, R_{2}\right)$ based on the top $m$ samples of $R$ and the corresponding samples of $\left(R_{1}, R_{2}\right)$. These estimates are $\hat{\rho}_{m, 10}=0.6356,0.4065,0.3892$, and $0.3414, \hat{\rho}_{m, 20}=$ $0.4520,0.1240,0.1173$, and 0.1628 , and $\hat{\rho}_{m, 12}=0.0537,-0.4704,-0.5281$, and -0.6054 for $m=10,000$ (all samples), 1,000, 500, and 100, respectively. They show that large system responses are weakly correlated with large modal responses, see correlation coefficients $\hat{\rho}_{m, k 0}$, $k=1,2$, and that modal responses corresponding to large system responses have either small and large values or moderate values since $\hat{\rho}_{m, 12}$ is negative for $m \leq 1,000$. The latter observation suggests that the the relationship between $R$ and $\left(R_{1}, R_{2}\right)$ has large uncertainty since large samples of $R$ are likely to be associated with large/small and small/large samples of $R_{1}$ and $R_{2}$. Fragilities defined as functions of $\left(R_{1}, R_{2}\right)$ will have large uncertainties.

Suppose now that $\left(R, R_{1}, R_{2}\right)$ is a Gaussian vector with mean $\left(\mu_{0}, \mu_{1}, \mu_{2}\right)$ and covariances $\gamma_{00}=E\left[\left(R-\mu_{0}\right)^{2}\right], \gamma_{k k}=E\left[\left(R_{k}-\mu_{0}\right)^{2}\right], \gamma_{0 k}=E\left[\left(R-\mu_{0}\right)\left(R_{k}-\mu_{k}\right)\right]$, and $\gamma_{12}=E\left[\left(R_{1}-\mu_{1}\right)\left(R_{2}-\mu_{2}\right)\right]=0$. This selection is consistent with the weak correlation between modal responses $R_{1}$ and $R_{2}$. The conditional random variables $R \mid\left(R_{1}, R_{2}\right)$ and $R \mid R_{k}$ are Gaussian with the properties

$$
\begin{aligned}
R \mid\left(R_{1}, R_{2}\right) & \sim N\left(\mu_{0}+\sum_{k=1}^{2} \frac{\gamma_{0 k}}{\gamma_{k k}}\left(R_{k}-\mu_{k}\right), \gamma_{00}-\sum_{k=1}^{2} \frac{\gamma_{0 k}^{2}}{\gamma_{k k}}\right) \\
R \mid R_{k} & \sim N\left(\mu_{0}+\frac{\gamma_{0 k}}{\gamma_{k k}}\left(R_{k}-\mu_{k}\right), \gamma_{00}-\frac{\gamma_{0 k}^{2}}{\gamma_{k k}}\right), \quad k=1,2 .
\end{aligned}
$$

Since $\operatorname{Var}\left[R \mid R_{1}, R_{2}\right] \leq \operatorname{Var}\left[R \mid R_{k}\right], k=1,2$, the vector-valued intensity measure $\left(R_{1}, R_{2}\right)$ is superior to the scalar measures $R_{k}, k=1,2$. The difference between $\operatorname{Var}\left[R \mid R_{1}, R_{2}\right]$ and $\operatorname{Var}\left[R \mid R_{k}\right]$ is the largest if $\gamma_{01}^{2} / \gamma_{k k} \simeq \gamma_{02}^{2} / \gamma_{k k}$ and is insignificant if one of modal responses is dominant. We note that Eq. 24 only provides qualitative information since the vector $\left(R, R_{1}, R_{2}\right)$ is not Gaussian.

The plots in Fig. 14 are similar to those in Fig. 13 except that they are for a stationary 

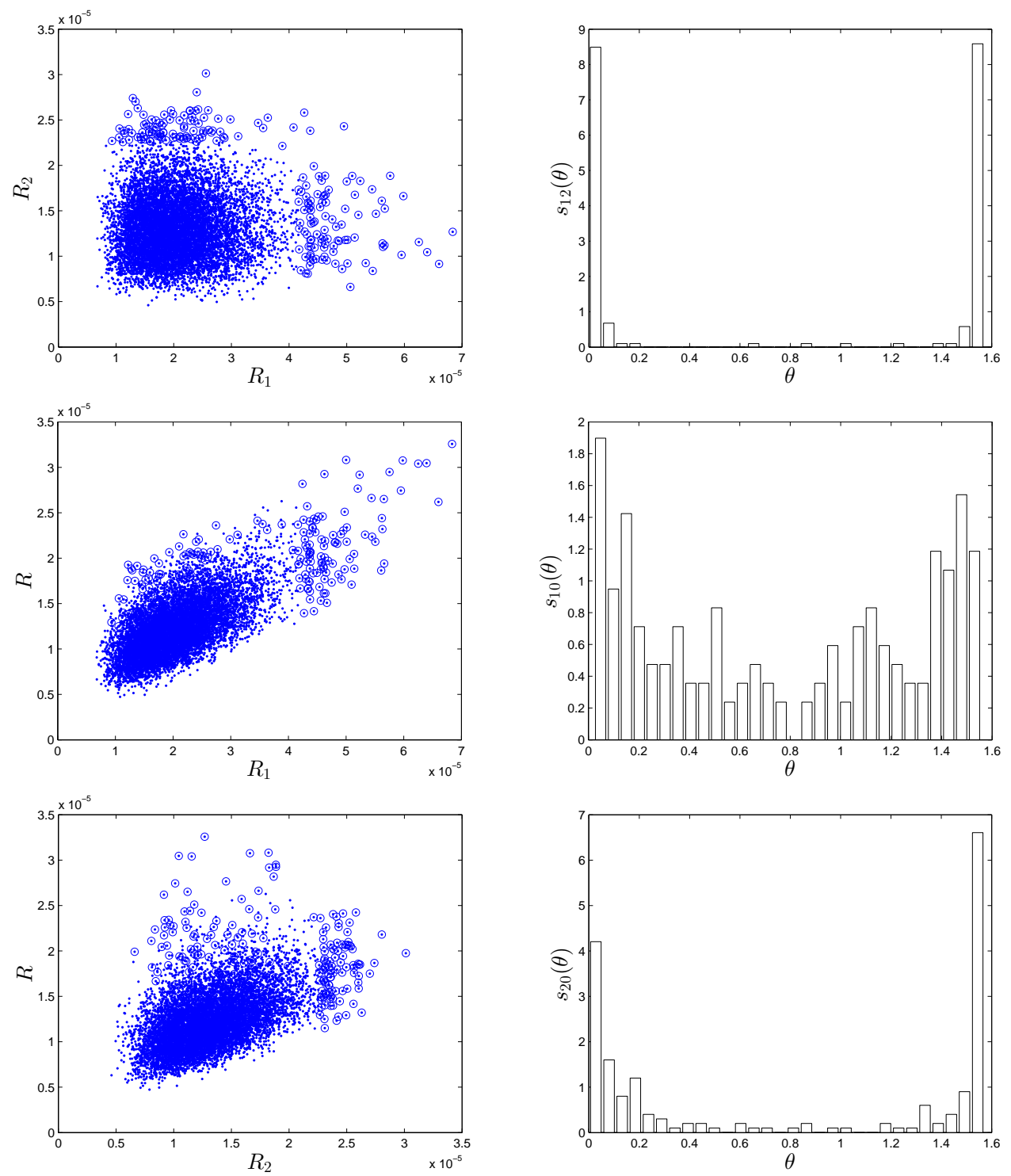

Figure 14: Samples of $\left(R_{1}, R_{2}\right),\left(R_{1}, R\right)$, and $\left(R_{2}, R\right)$ (left panels) and angular measures $s_{12}(\theta), s_{10}(\theta)$, and $s_{20}(\theta)$ of $\left(R_{1}, R_{2}\right),\left(R_{1}, R\right)$, and $\left(R_{2}, R\right)$ (right panels) for $\alpha=0.4$ and a $\operatorname{SBM} A(t)$

Gaussian process $A(t)$ with mean zero and spectral density in the left panel of Fig. 5 given by the specific barrier model (SBM) and a factor $\alpha=0.4$ defining modal contributions rather than $\alpha=0.3$. As in Fig. 13, the 100 samples marked with circles have been used to estimate the angular measures of $\left(R_{1}, R_{2}\right),\left(R, R_{1}\right)$, and $\left(R, R_{2}\right)$. These measures are shown in the right panels of Fig. 14. We note that simultaneously large values of $R_{1}$ and $R_{2}$ are unlikely and the dependence between large values of $R$ and $R_{1}$ is stronger than that between large values of $R$ and $R_{2}$. As for the BLWN ground motion $A(t)$, large response samples are associated with samples of $R_{1}$ and $R_{2}$ or, equivalently, samples of $S_{a}\left(T_{1}\right)$ and $S_{a}\left(T_{2}\right)$, which are negatively correlated, e.g., $\hat{\rho}_{m, 12}=-0.6184$ and -0.4221 for the top 188 and 1736 samples of $R$. 


\section{Conclusions}

Fragilities are frequently defined as functions of single/multiple ordinates of the pseudoacceleration response spectrum $S_{a}(T)$ and used extensively as intensity measures (IMs) in performance-based earthquake engineering. A critical assumption of this approach is that $S_{a}(T)$ captures sufficient information on the seismic ground acceleration process such that demand parameters $D$ of nonlinear, complex, multi-degree of freedom structures correlate satisfactory with single/multiple ordinates of $S_{a}(T)$. If this assumption is invalid, resulting fragilities will provide limited if any information on structural performance.

This study has examined the validity of the assumption that the dependence between $S_{a}(T)$ and $D$ is adequate for fragility analysis. It was shown that (1) demand parameters $D$ for simple nonlinear structures, e.g., a Bouc-Wen structure with a single degree of freedom, and ordinates of $S_{a}(T)$ are weakly dependent and (2) demand parameters $D$ for linear multidegree of freedom structures with proportional damping and two or more contributing modes and multiple ordinates of $S_{a}(T)$ are weakly dependent. It was concluded that fragilities defined as functions of single/multiple ordinates of $S_{a}(T)$ can have large uncertainties which limits their usefulness. Correlation coefficients, copula models, and concepts of multivariate extreme value theory have been used to quantify the dependence between $S_{a}(T)$ and $D$.

\section{Acknowledgements}

The work reported in this paper has been supported by the National Science Foundation under grand CMMI-095714 and CMMI-1639669. This support is gratefully acknowledged.

\section{References}

[1] A. AghaKouchak, D. Easterling, K. Hsu, S. Schubert, and S. Sorooshian, editors. Extremes in a Changing Climate. Detection, Analysis and Uncertainty. Water Science and Technology Library. Springer, Dordrecht, Heidelberg, New York, London, 2013.

[2] J. W. Baker and C. A. Cornell. A vector-valued ground motion intenxity measure consisiting of spectral acceleration and epsilon. Earthquake Engineering and Structural Dynamics, 34:1193-1217, 2005.

[3] W. Baker, J. Conditional mean spectrum: Tool for ground-motion selection. Journal of Structural Engineering, 137:322-331, 2011.

[4] H. Ebrahimian, F. Jalayer, A. Lucchini, F. Mollaioli, and G. Manfredi. Preliminary ranking of alternative scalar and vector intensity measures of ground shaking. Bulletin of Earthquake Engineering, 2015. DOI 10.1007/s10518-015-9755-9.

[5] C. Genest and A-C. Favre. Everything you always wanted to know about copula modeling but were afraid to ask. Journal of Hydrologic Engineering, 12(4):347-368, 2007.

[6] C. Genest and J. Segers. Rank-based inference for bivariate extreme-value copulas. The Annals of Statistics, 37:2990-3022, 2009. 
[7] M. Grigoriu. Mean-square structural response to stationary ground acceleration. Journal of the Engineering Mechanics Division, 107:969-986, 1981.

[8] M. Grigoriu. Applied Non-Gaussian Processes: Examples, Theory, Simulation, Linear Random Vibration, and MATLAB Solutions. Prentice Hall, Englewoods Cliffs, NJ, 1995.

[9] M. Grigoriu. Stochastic Calculus. Applications in Science and Engineering. Birkhäuser, Boston, 2002.

[10] C. Kafali and M. Grigoriu. Seismic fragility analysis: Application to simple linear and nonlinear systems. Earthquake Engineering and Structural Dynamics, 36:1885-1900, 2007.

[11] Chopra A. K. Kwong, N. S. and R. K. McGuire. Evaluation of ground motion selection and modification procedures using synthetic ground motions. Earthquake Engineering and Structural Dynamics, 44:1841-1861, 2015. DOI: 10.1002/eqe.2558.

[12] Chopra A. K. Kwong, N. S. and R. K. McGuire. A framework for the evaluation of ground motion selection and modification procedures. Earthquake Engineering and Structural Dynamics, 44:795-815, 2015. DOI: 10.1002/eqe.2502.

[13] Chopra A. K. Kwong, N. S. and R. K. McGuire. A ground motion selection procedure for enforcing hazard consistency and estimating seismic demand hazard curves. Earthquake Engineering and Structural Dynamics, 44:24672487, 2015. DOI: 10.1002/eqe.2593.

[14] M. R. Leadbetter, G. Lindgren, and H. Rootzén. Extremes and Related Properties of Random Sequences and Processes. Springer-Verlag, New York, 1983.

[15] Th. Mikosch. Copulas: Tails and facts-rejoinder. Extremes, 9:3-20, 2006 . DOI 10.1007/s10687-006-0015-x.

[16] A. S. Papageorgiou and K. Aki. A specific barrier model for the quantitative description of the inhomogeneous faulting and the prediction of strong ground motion. Part I: Description of the model. Bulletin of the Seismilogical Society of America, pages 693$722,1983$.

[17] S. Resnick. On the foundations of multivariate heavy-tail analysis. Journal of Applied Probability, 41:191-212, 2004.

[18] S. Resnick. Heavy-Tail Phenomena: Probabilistic and Statistical Modeling. Springer, New York, 2007.

[19] T. T. Soong and M. Grigoriu. Random Vibration of Mechanical and Structural Systems. Prentice Hall, Englewood Cliffs, N.J., 1993. 